\title{
Spectral action and the electroweak $\theta$-terms for the Standard Model without fermion doubling
}

\section{A. Bochniak, A. Sitarz and P. Zalecki}

\author{
Institute of Theoretical Physics, Jagiellonian University, \\ prof. Stanisława Eojasiewicza 11, Kraków 30-348, Poland \\ E-mail: arkadiusz.bochniak@doctoral.uj.edu.pl, \\ andrzej.sitarz@uj.edu.pl, pawel.zalecki@doctoral.uj.edu.pl
}

ABSTRACT: We compute the leading terms of the spectral action for a noncommutative geometry model that has no fermion doubling. The spectral triple describing it, which is chiral and allows for CP-symmetry breaking, has the Dirac operator that is not of the product type. Using Wick rotation we derive explicitly the Lagrangian of the model from the spectral action for a flat metric, demonstrating the appearance of the topological $\theta$ terms for the electroweak gauge fields.

KEYwords: Non-Commutative Geometry, Gauge Symmetry, CP violation, Quark Masses and SM Parameters

ArXiv EPrint: 2106.10890 


\section{Contents}

1 Introduction 1

2 The starting point: fermions and the algebra of the Standard Model 2

2.1 The gauge transformations and the Higgs 4

3 The spectral action $\quad 7$

3.1 Spectral action - physical parametrization 8

3.2 The spectral action for the full static SM 9

$\begin{array}{ll}3.2 .1 \text { The full spectral action } & 10\end{array}$

$\begin{array}{lll}3.3 & \text { Wick rotated model } & 11\end{array}$

$\begin{array}{lll}3.3 .1 & \text { The full spectral action } & 12\end{array}$

4 Conclusions and outlook $\quad 14$

$\begin{array}{lr}\text { A The static spectral action } & \mathbf{1 5}\end{array}$

$\begin{array}{lll}\text { A.1 Leptonic sector } & 15\end{array}$

$\begin{array}{ll}\text { A.2 Quark sector } & 17\end{array}$

$\begin{array}{ll}\text { B The Wick rotated model } & 19\end{array}$

$\begin{array}{ll}\text { B.1 Leptonic sector } & 19\end{array}$

$\begin{array}{lll}\text { B.2 Quark sector } & 22\end{array}$

\section{Introduction}

The Standard Model of Particle Physics is a powerful theory that gives a precise and effective description of all fundamental forces apart from gravity. Its predictive power and agreement with experimental results guarantee that it needs to remain the backbone of any fundamental theory of particle interactions. Yet, in contrast to General Relativity, which is deeply rooted in the geometry of space-time, the Standard Model only partially can be explained similarly. The structure of gauge theory and the Yang-Mills action signifies that indeed the geometry plays there a significant role. However, the appearance of a Higgs field and the symmetry-breaking quartic potential are not directly implied by classical geometry.

The hint that the Standard Model has a more subtle structure came from noncommutative geometry and the theory of spectral triples. Founded by Alain Connes to solve significant mathematical problems related to the index theorem of Atiyah and Singer (see [1] for a review), the theory is a well-structured non-trivial generalization of classical differential geometry that allows studying not only differentiable manifolds but also discrete spaces, fractals and quantum deformations of spaces from a novel point of view. Interestingly, the tools of noncommutative geometry allowed to construct models that explain 
the geometry of the Standard Model [2-4] (see also [5] and [6] for detailed discussion) and its extensions [7-11]. Their structure is similar to Kaluza-Klein models yet with a finite noncommutative algebra instead of the additional dimension of space-time. The geometry of the entire enhanced space-time is determined by a Dirac operator that depends on the metric and the gauge connections, and also includes the Higgs field, which plays a role of a connection over the finite noncommutative component. The spectral action then gives the full gravity and Yang-Mills action with the quartic Higgs potential and minimal couplings between the Higgs and the gauge fields [12].

The story of the noncommutative model-building is, however, not yet complete as the most accepted model is in the Euclidean signature and requires additional assumptions to remove the possibility of the $\mathrm{SU}(3)$ symmetry breaking $[13,14]$ as well as an additional projection onto the physical space of fermions (due to the fermion quadrupling in the model) [15-17]. In the analysis of the Lorentzian case with slight modifications of the spectral triple rules we proved that there exists a model without the fermion doubling and with exact colour SU(3) symmetry [18]. Moreover, the non-product Dirac operator satisfied a slightly modified first-order condition which is tantamount to the $\operatorname{spin}_{c}$ one under certain requirements for mass spectra of fermions. The CP-symmetry breaking in the Standard Model was then geometrically explained as the lack of reality symmetry of the finite component of the Dirac operator as witnessed by nonvanishing of the Wolfenstein parameter and the CP-phase in the neutrino sector.

In the paper, we compute the spectral action for the model we presented in [18]. It needs to be stressed that this model is not of the product-type geometry and therefore the computations and results are not automatically identical to those performed in the series of papers computing the spectral action $[12,19]$. In addition, as we start with the Lorentzian model we need to perform a Wick rotation to be able to use heat trace techniques [20] or restrict the model to spatial and time-independent (static) components of the fields. To check the consistency of the computations we perform both operations. The new element of the spectral action, apart from slight differences in the relative coefficients, is the appearance of topological theta terms for the gauge fields in the electroweak sector. This is a characteristic new feature of this model, which is inherently chiral, especially that such terms cannot appear in the spectral action of the product geometries.

\section{The starting point: fermions and the algebra of the Standard Model}

We begin by briefly reviewing the model as described in details in $[18,22]$. The particle content in the one-generation Standard Model can be conveniently parametrized in the following form:

$$
\Psi=\left(\begin{array}{cccc}
\nu_{R} & u_{R}^{1} & u_{R}^{2} & u_{R}^{3} \\
e_{R} & d_{R}^{1} & d_{R}^{2} & d_{R}^{3} \\
\nu_{L} & u_{L}^{1} & u_{L}^{2} & u_{L}^{3} \\
e_{L} & d_{L}^{1} & d_{L}^{2} & d_{L}^{3}
\end{array}\right) \in M_{4}\left(H_{W}\right)
$$


Every entry of the above matrix is a Weyl spinor (from $H_{W}$ ) over the Minkowski space $\mathcal{M}^{1,3}$. The algebra $\mathcal{A}$ is taken to consist of (smooth) $\mathbb{C} \oplus \mathbb{H} \oplus M_{3}(\mathbb{C})$-valued functions over $\mathcal{M}^{1,3}$. We choose its left and right real representations:

$$
\pi_{L}(\lambda, q, m) \Psi=\left(\begin{array}{ccc}
\lambda & & \\
& \bar{\lambda} & \\
& & q
\end{array}\right) \Psi, \quad \pi_{R}(\lambda, q, m) \Psi=\Psi\left(\begin{array}{ll}
\bar{\lambda} & \\
& m^{\dagger}
\end{array}\right),
$$

where $\lambda, q$ and $m$ are complex, quaternion and $M_{3}(\mathbb{C})$-valued functions, respectively. Since left and right multiplications commute, the zeroth-order condition is satisfied, i.e.

$$
\left[\pi_{L}(a), \pi_{R}(b)\right]=0
$$

for all $a, b \in \mathcal{A}$. It is convenient to encode local linear operator acting on the particle content of the model, at every point of $\mathcal{M}^{1,3}$, as an element of $M_{4}(\mathbb{C}) \otimes M_{2}(\mathbb{C}) \otimes M_{4}(\mathbb{C})$, where the first and the last matrices act by multiplication from the left and from the right, respectively, while the middle $M_{2}(\mathbb{C})$ matrix acts on the components of the Weyl spinor. For the algebra $\mathcal{A}$ this component is, of course, identity matrix.

Using this notation, the full Lorentzian Dirac operator of the Standard Model can be written of the form,

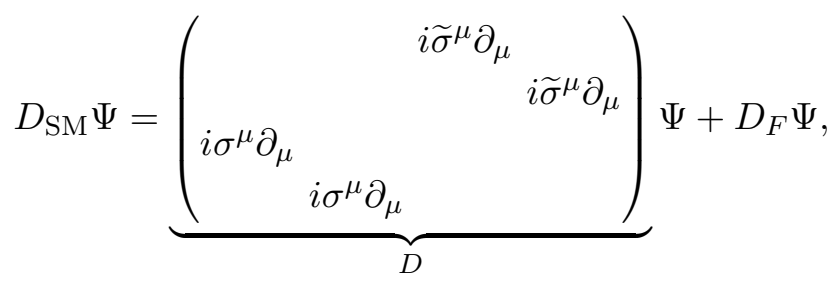

where $\sigma^{0}=\mathbf{1}_{2}=\widetilde{\sigma}^{0}$ and $\widetilde{\sigma}^{i}=-\sigma^{i}$, the latter being standard Pauli matrices. $D_{F}$ is a finite endomorphism of the Hilbert space $M_{4}\left(H_{W}\right)$.

In [18] the Krein-shifted full Dirac operator of the Standard Model, $\widetilde{D_{\mathrm{SM}}}=\beta D_{\mathrm{SM}}$, where

$$
\beta=\left(\begin{array}{l}
\mathbf{1}_{2} \\
\mathbf{1}_{2}
\end{array}\right) \otimes \mathbf{1}_{2} \otimes \mathbf{1}_{4}
$$

was discussed in details. The Krein-shifted manifold component of the Lorentzian Dirac operator $\widetilde{D}$ in the local Cartesian coordinates over $\mathbb{R}^{4}$, with a flat metric, is

$$
\widetilde{D}=\left(\begin{array}{cc}
\mathbf{1}_{2} & \\
& \mathbf{0}_{2}
\end{array}\right) \otimes i \sigma^{\mu} \partial_{\mu} \otimes \mathbf{1}_{4}+\left(\begin{array}{ll}
\mathbf{0}_{2} & \\
& \mathbf{1}_{2}
\end{array}\right) \otimes i \widetilde{\sigma}^{\mu} \partial_{\mu} \otimes \mathbf{1}_{4},
$$

whereas the Krein-shifted discrete part of the Dirac operator is,

$$
\widetilde{D_{F}}=\underbrace{\left(\begin{array}{c}
M_{l} \\
M_{l}^{\dagger}
\end{array}\right)}_{D_{l}} \otimes \mathbf{1}_{2} \otimes e_{11}+\underbrace{\left(\begin{array}{c}
M_{q} \\
M_{q}^{\dagger}
\end{array}\right)}_{D_{q}} \otimes \mathbf{1}_{2} \otimes\left(\mathbf{1}_{4}-e_{11}\right),
$$

where $M_{l}, M_{q} \in M_{2}(\mathbb{C})$. 
The Krein-shifted Dirac operator and the algebra were proven to satisfy the generalized order-one condition, that is for all $a, b \in A$,

$$
\left.\left[\pi_{R}(a),\left[\widetilde{D_{\mathrm{SM}}}, \pi_{L}(b)\right]\right]=0, \quad\left[\pi_{L}(a), \widetilde{\left[D_{\mathrm{SM}}\right.}, \pi_{R}(b)\right]\right]=0 .
$$

Note that although the usual order-one condition, which is implemented with the real structure, can also be written in this form, the above generalized version extends it to the case of Riemannian manifolds, which are not spin [23].

The Lorentzian spectral triple for the signature $(1,3)$ has the standard chirality $\mathbb{Z}_{2^{-}}$ grading $\gamma$ and the charge conjugation operator, $\mathcal{J}$,

$$
\gamma=\left(\begin{array}{cc}
1_{2} & 0 \\
0 & -1_{2}
\end{array}\right), \quad \mathcal{J}=i \gamma^{2} \circ c c=i\left(\begin{array}{cc}
0 & \sigma^{2} \\
-\sigma^{2} & 0
\end{array}\right) \circ c c,
$$

where $c c$ denotes the usual complex conjugation of spinors. The construction can be easily generalized for the three families of leptons and quarks by enlarging the Hilbert space diagonally, i.e. by taking $M_{4}\left(H_{W}\right) \otimes \mathbb{C}^{3}$ with the diagonal representation of the algebra. The matrices $M_{l}$ and $M_{q}$ in $(2.5)$ are no longer in $M_{2}(\mathbb{C})$ but in $M_{2}(\mathbb{C}) \otimes M_{3}(\mathbb{C})$. Its standard presentation for the physical Standard Model is

$$
M_{l}=\left(\begin{array}{cc}
\Upsilon_{\nu} & 0 \\
0 & \Upsilon_{e}
\end{array}\right), \quad M_{q}=\left(\begin{array}{cc}
\Upsilon_{u} & 0 \\
0 & \Upsilon_{d}
\end{array}\right)
$$

where $\Upsilon_{e}$ and $\Upsilon_{u}$ are chosen diagonal with the masses of electron, muon, and tau and the up, charm, and top quarks, respectively, and $\Upsilon_{\nu}$ and $\Upsilon_{d}$ can be diagonalised by the Pontecorvo-Maki-Nakagawa-Sakata mixing matrix (PMNS matrix) $U$ and the CabibboKobayashi-Maskawa mixing matrix (CKM matrix) $V$, respectively:

$$
\Upsilon_{\nu}=U \widetilde{\Upsilon_{\nu}} U^{\dagger}, \quad \Upsilon_{d}=V \widetilde{\Upsilon_{d}} V^{\dagger}
$$

The matrices $\widetilde{\Upsilon_{\nu}}, \widetilde{\Upsilon_{d}}$ provide (Dirac) masses of all neutrinos and down, strange, and bottom quarks.

As it was demonstrated in [18] the model has interesting algebraic properties, like the Morita duality (which means that the generalized Clifford algebra is a full commutant of the algebra $\mathcal{A})$ provided that both pairs of matrices $\left(\Upsilon_{\nu}, \Upsilon_{e}\right)$ and $\left(\Upsilon_{u}, \Upsilon_{d}\right)$ have pairwise different eigenvalues. Furthermore, without referring to additional symmetries or assumptions the model preserves the SU(3) strong symmetry and allows for the natural breaking of the CP-symmetry, which is linked to the non-reality of the mixing matrices. This is, on the level of the algebra of the model, equivalent to the failure of the finite part of the Krein-shifted Dirac operator to be $\mathcal{J}$-real (see [18] for details).

\subsection{The gauge transformations and the Higgs}

In this section we extend the model by introducing the fluctuations of the Dirac operator, that is a family of operators obtained from $\widetilde{D_{\mathrm{SM}}}$ by perturbing them with one-forms, that originate from commutators with the Dirac operator. Here, both left and right representations will contribute to the gauge transformations and the fluctuations of the Dirac operator. 
A gauge transformation of physical fields is a unitary transformation of the form,

$$
U_{L R}=\pi_{L}(U) \pi_{R}(U)
$$

for a unitary element $U$ of the algebra $\mathcal{A}$, so that the gauge-transformed Dirac operator becomes:

$$
{\widetilde{D_{\mathrm{SM}}}}^{U}=\pi_{L}(U) \pi_{R}(U) \widetilde{D_{\mathrm{SM}}} \pi_{R}\left(U^{\dagger}\right) \pi_{L}\left(U^{\dagger}\right),
$$

which, after using the order-zero and order-one conditions, can be rewritten as

$$
\widetilde{D_{\mathrm{SM}}} U=\widetilde{D_{\mathrm{SM}}}+\pi_{L}(U)\left[\widetilde{D_{\mathrm{SM}}}, \pi_{L}\left(U^{\dagger}\right)\right]+\pi_{R}(U)\left[\widetilde{D_{\mathrm{SM}}}, \pi_{R}\left(U^{\dagger}\right)\right]
$$

The unitary group of the finite algebra is $\mathrm{U}(1) \times \mathrm{SU}(2) \times \mathrm{U}(3)$, while the unitaries of the form $U_{L R}$ are elements of the group being a quotient of this group by its diagonal normal $\operatorname{subgroup} \mathbb{Z}_{2}=\left\{ \pm\left(1, \mathbf{1}_{2}, \mathbf{1}_{3}\right)\right\}$.

To parametrize the fluctuations we first start with computing left and right ones separately:

$$
\sum_{j} \pi_{L}\left(a_{j}\right)\left[\widetilde{D_{\mathrm{SM}}}, \pi_{L}\left(b_{j}\right)\right], \quad \sum_{j} \pi_{R}\left(a_{j}\right)\left[\widetilde{D_{\mathrm{SM}}}, \pi_{R}\left(b_{j}\right)\right],
$$

where $a_{j}, b_{j} \in \mathcal{A}=C^{\infty}\left(\mathbb{R}^{4}, \mathbb{C} \oplus \mathbb{H} \oplus M_{3}(\mathbb{C})\right)$, and the representations $\pi_{L}$ and $\pi_{R}$ are of the form:

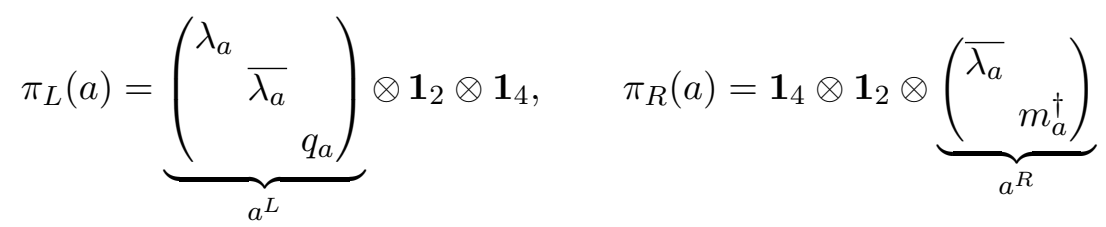

where $\lambda_{a} \in C^{\infty}\left(\mathbb{R}^{4}\right), q_{a} \in C^{\infty}\left(\mathbb{R}^{4}, \mathbb{H}\right)$ and $m_{a} \in C^{\infty}\left(\mathbb{R}^{4}, M_{3}(\mathbb{C})\right)$.

We first notice that $\left[\widetilde{D_{F}}, \pi_{R}(b)\right]=0$ from the very definition of the representation and the structure of this Dirac operator. Therefore, the only contribution from the right fluctuations can be parametrized as

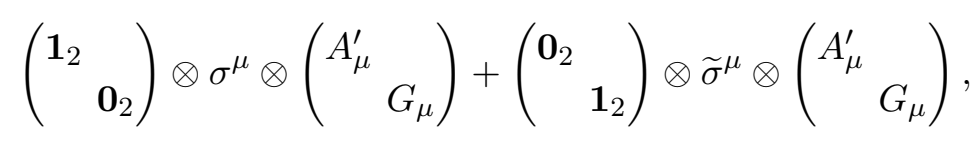

where $A_{\mu}^{\prime}=i \sum_{j} \overline{\lambda_{a_{j}}}\left(\partial_{\mu} \overline{\lambda_{b_{j}}}\right)$ and $G_{\mu}=i \sum_{j} m_{a_{j}}^{\dagger}\left(\partial_{\mu} m_{b_{j}}^{\dagger}\right)$.

Now, we compute the left fluctuations. Starting with the ones following from the $\widetilde{D}$ part of the Dirac operator we immediately get

$$
\sum_{j} \pi_{L}\left(a_{j}\right)\left[\widetilde{D}, \pi_{L}\left(b_{j}\right)\right]=A_{\mu}^{R} \otimes \sigma^{\mu} \otimes \mathbf{1}_{4}+A_{\mu}^{L} \otimes \widetilde{\sigma}^{\mu} \otimes \mathbf{1}_{4},
$$

with

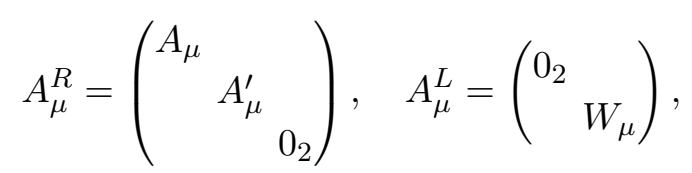


where $A_{\mu}=i \sum_{j} \lambda_{a_{j}}\left(\partial_{\mu} \lambda_{b_{j}}\right), A_{\mu}^{\prime}$ is as previously, and $W_{\mu}=i \sum_{j} q_{a_{j}}\left(\partial_{\mu} q_{b_{j}}\right)$. Here $A_{\mu}$ and $A_{\mu}^{\prime}$ do not describe the single (electromagnetic) $\mathrm{U}(1)$ gauge field, but contain also the $Z$ boson counterpart.

Imposing the selfadjointness condition we immediately get $A_{\mu}^{\prime}=-A_{\mu}$ and infer that $W_{\mu}$ is indeed an element of $i \mathfrak{s u}(2)$ (as it is enforced to be a real linear combination of Pauli matrices). Similarly, we deduce that $G_{\mu}$ is a $\mathrm{U}(3)$ gauge field.

It remains to take into account the contribution from $\widetilde{D_{F}}$. By a straightforward computation we get

$$
\sum_{j} \pi_{L}\left(a_{j}\right)\left[\widetilde{D_{F}}, \pi_{L}\left(b_{j}\right)\right]=\phi^{l} \otimes 1_{2} \otimes e_{11}+\phi^{q} \otimes 1_{2} \otimes\left(1_{4}-e_{11}\right)
$$

where

$$
\phi^{r}=\sum_{j} a_{j}^{L}\left[D_{r}, b_{j}^{L}\right], \quad r=l, q .
$$

Since both matrices $M_{l}$ and $M_{q}$ are diagonal, they commute with $\operatorname{diag}(\lambda, \bar{\lambda})$. Denoting by

$$
\mathbf{\Phi}=\sum_{j}\left({ }^{\lambda_{a_{j}}} \frac{}{\lambda_{a_{j}}}\right)\left[q_{b_{j}}-\left({ }^{\lambda_{b_{j}}} \frac{}{\lambda_{b_{j}}}\right)\right],
$$

we can parametrize those fluctuations, under the assumption of selfadjointness, as:

$$
\left(\boldsymbol{\Phi}^{\dagger} M_{l}^{\dagger} M_{l} \boldsymbol{\Phi}\right) \otimes \mathbf{1}_{2} \otimes e_{11}+\left({\boldsymbol{\Phi}^{\dagger} M_{q}^{\dagger}}^{M_{q} \boldsymbol{\Phi}}\right) \otimes \mathbf{1}_{2} \otimes\left(\mathbf{1}_{4}-e_{11}\right) .
$$

As a result, the fluctuations coming from all the terms can be parametrize as

$$
\begin{aligned}
& \omega=A_{\mu} e_{11} \otimes \sigma^{\mu} \otimes\left(\mathbf{1}_{4}-e_{11}\right)-2 A_{\mu} e_{22} \otimes \sigma^{\mu} \otimes e_{11} \\
& -A_{\mu} e_{22} \otimes \sigma^{\mu} \otimes\left(\mathbf{1}_{4}-e_{11}\right)-A_{\mu}\left(e_{33}+e_{44}\right) \otimes \tilde{\sigma}^{\mu} \otimes e_{11} \\
& +\left(\begin{array}{ll}
\mathbf{0}_{2} & \\
& W_{\mu}
\end{array}\right) \otimes \widetilde{\sigma}^{\mu} \otimes \mathbf{1}_{4} \\
& +\left(\begin{array}{cc}
\mathbf{1}_{2} & \\
& \mathbf{0}_{2}
\end{array}\right) \otimes \sigma^{\mu} \otimes\left(\begin{array}{ll}
\mathbf{0}_{1} & \\
& G_{\mu}
\end{array}\right)+\left(\begin{array}{ll}
\mathbf{0}_{2} & \\
& \\
& \mathbf{1}_{2}
\end{array}\right) \otimes \widetilde{\sigma}^{\mu} \otimes\left(\begin{array}{ll}
\mathbf{0}_{1} & \\
& G_{\mu}
\end{array}\right) \\
& +\left({ }_{\boldsymbol{\Phi}^{\dagger} M_{l}^{\dagger}}^{M_{l} \boldsymbol{\Phi}}\right) \otimes \mathbf{1}_{2} \otimes e_{11}+\left({ }_{\boldsymbol{\Phi}^{\dagger} M_{q}^{\dagger}} M_{q} \boldsymbol{\Phi}\right) \otimes \mathbf{1}_{2} \otimes\left(\mathbf{1}_{4}-e_{11}\right) .
\end{aligned}
$$

We denote the fluctuated Dirac operator by $\widetilde{D_{\mathrm{SM}}} \omega=\widetilde{D_{\mathrm{SM}}}+\omega$.

For a unitary element $U \equiv\left(u_{1}, u_{2}, u_{3}\right) \in \mathrm{U}(1) \times \mathrm{SU}(2) \times \mathrm{U}(3)$ the gauge-transformed fluctuated Dirac operator is of the form

$$
{\widetilde{D_{\mathrm{SM}}}}^{\omega}=\pi_{L}(U) \pi_{R}(U){\widetilde{D_{\mathrm{SM}}}}^{\omega} \pi_{R}\left(U^{\dagger}\right) \pi_{L}\left(U^{\dagger}\right) .
$$

The gauge transformation can be therefore implemented by

$$
\begin{aligned}
\omega \longmapsto \omega^{U}= & \pi_{L}(U) \pi_{R}(U) \omega \pi_{R}\left(U^{\dagger}\right) \pi_{L}\left(U^{\dagger}\right) \\
& +\pi_{L}(U)\left[\widetilde{D_{\mathrm{SM}}}, \pi_{L}\left(U^{\dagger}\right)\right]+\pi_{R}(U)\left[\widetilde{D_{\mathrm{SM}}}, \pi_{R}\left(U^{\dagger}\right)\right] .
\end{aligned}
$$


As a result, the fields $A_{\mu}, W_{\mu}, G_{\mu}, \boldsymbol{\Phi}$ transform accordingly:

$$
\begin{aligned}
A_{\mu} & \longmapsto A_{\mu}+u_{1}\left(\partial_{\mu} \overline{u_{1}}\right), \\
W_{\mu} & \longmapsto u_{2} W_{\mu} u_{2}^{\dagger}+u_{2}\left(\partial_{\mu} u_{2}^{\dagger}\right), \\
G_{\mu} & \longmapsto u_{3} G_{\mu} u_{3}^{\dagger}+u_{3}\left(\partial_{\mu} u_{3}^{\dagger}\right), \\
\mathbf{1}_{2}+\boldsymbol{\Phi} & \longmapsto\left(\begin{array}{c}
u_{1} \\
\overline{u_{1}}
\end{array}\right)\left(\mathbf{1}_{2}+\mathbf{\Phi}\right) u_{2}^{\dagger} .
\end{aligned}
$$

We remark that in the above derivation the crucial role was played by the fact that the representation of $\mathrm{U}(1)$ part of the gauge group commutes with the mass and mixing matrices.

It is known that the gauge group of the Standard Model should contain SU(3) rather than $\mathrm{U}(3)$. This can be achieved with a further condition, the unimodularity of the representation, which, however, can be imposed in different ways. In particular, let us observe that the left action of the group is unimodular from the beginning. For the right representation, one could require either the condition that it is unimodular on each fundamental component (chiral lepton and quark) or in the full representation, including all chiral fermions and families. In the first case, the unimodularity condition is equivalent to $u_{1} \operatorname{det} u_{3}=1$, whereas in the second case it becomes $\left(u_{1} \operatorname{det} u_{3}\right)^{12}=1$. In the first case, the resulting group is exactly the group of the Standard Model,

$$
(\mathrm{U}(1) \times \mathrm{SU}(2) \times \mathrm{SU}(3)) / \mathbb{Z}_{6},
$$

whereas in the latter case it is the one described in [6, proposition 11.4], which differs from the gauge group of the Standard Model by a finite factor. Independently, their Lie algebras agree and the finite difference does not affect the structure of the gauge fields. The unimodularity condition on the Lie algebra, instead of the Lie group, level of perturbation means that the trace of a perturbation has to vanish. This condition results in $\operatorname{Tr}\left(G_{\mu}\right)=A_{\mu}$. We therefore introduce the traceless gauge field $G_{\mu}^{\prime}=G_{\mu}-\frac{1}{3} A_{\mu} \mathbf{1}_{3}$ and then in the perturbations we can replace $G_{\mu}$ by $G_{\mu}^{\prime}+\frac{1}{3} A_{\mu} \mathbf{1}_{3}$, where now $G_{\mu}^{\prime}$ is assumed to be a $\mathrm{SU}(3)$ gauge field. By a slight abuse of notation we will use $G_{\mu}$ instead of $G_{\mu}^{\prime}$ in the rest of the paper.

\section{The spectral action}

The spectral action, as defined originally by Chamseddine and Connes [24], makes sense for elliptic operators on Euclidean manifolds and, in the noncommutative generalisation, for spectral triples. To make contact with physics the usual method is to compute the spectral action in the Euclidean setup and then to Wick-rotate it to the Lorentzian signature. Yet this procedure starts directly from the Euclidean formulation of the model, which may not be equivalent to the Lorentzian. On the other hand, it is feasible to start with the genuine Lorentzian spectral triple and then look either for the appropriate spectral action principle (the first steps towards it have already been done in [25]) or use the Wick-rotated Lorentzian operator (so that then we can work with an elliptic operator for which the 
spectral action is computable) and then Wick-rotate the result back to the Lorentzian case. It remains an interesting general question whether both procedures give the same result. Since the results of [25] have not been so far extended to Dirac-type operators, we proceed with the latter procedure, however, to have another check of the result we compute the spectral action of "static and spatial" part of the Dirac operator, which is an elliptic operator (we explain the procedure in subsection 3.2). Finally, let us remark that we perform computations on a flat manifold, which could be taken as a compact 4-torus, however, since all Gilkey-Seeley-DeWitt coefficients are local the results are extendable to the physical action on a Minkowski space.

In the considerations so far (see e.g. [6] and references therein), the spectral action for the Standard Model was computed for the Euclidean model with fermion doubling. Moreover, the assumed bare Dirac operator was of the product type and therefore its square was simply the sum of the squares of the Dirac operators on manifold component and on the discrete component. While this strategy can be justified by the arguments of covariance and geometric character of the action, the relative coefficients and the proportions between them may in general differ, when one considers the Lorentzian and explicitly chiral Dirac operator.

Of course, the best strategy would be to apply a genuine Lorentzian approach (see [25]), however, this appears to be at the moment restricted only to scalar operators and not Diractype operators. Therefore we propose two simple, computable methods to obtain an insight into the action of the model, which is motivated by spectral methods.

The first one assumes that we restrict ourselves to the static and spatial case, computing the terms of the spectral action for the Krein-shifted Dirac operator that is restricted to the spatial part and with the gauge fields that arise exclusively through static (timeindependent) gauge transformations. Such restricted Dirac operator is already a hermitian elliptic operator and one can easily compute the heat trace coefficients of its square. This shall recover the action of the model for the time-independent fields, which is invariant under static gauge transformations. However, one cannot expect that all terms of the action will be present, and their coefficients correct.

The second method takes as the input the Wick-rotated Lorentzian Dirac operator $D_{w}$. Such operator is elliptic, as its continuous part is just the usual Wick-rotated Dirac operator (with gauge fields) over the flat space-time. However, the discrete part of the operator (which is not Krein-shifted) is alone not hermitian but only normal. Nevertheless one can still compute the heat trace coefficients of $D_{w}^{\dagger} D_{w}$ and then, using the Wick rotation back to the Lorentzian case recover the action functional.

In what follows we assume that we work on a flat compact manifold (torus) so all curvature terms vanish from the spectral action, and we are using a physical parametrisation of fields, described next.

\subsection{Spectral action - physical parametrization}

Let us now write explicitly the full spectral action in terms of Yukawa parameters and Higgs field in the case of one generation of fermions. Since $\boldsymbol{\Phi}$ is a quaternionic field it can 
be parametrize as

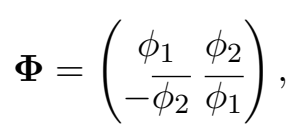

where $\phi_{1}, \phi_{2}$ are two complex fields. Then

$$
\begin{gathered}
\Phi_{l}=M_{l}(1+\boldsymbol{\Phi})=\left(\begin{array}{cc}
\Upsilon_{\nu} H_{1} & \Upsilon_{\nu} H_{2} \\
-\Upsilon_{e} \overline{H_{2}} & \Upsilon_{e} \overline{H_{1}}
\end{array}\right), \\
\Phi_{q}=M_{q}(1+\boldsymbol{\Phi})=\left(\begin{array}{cc}
\Upsilon_{u} H_{1} & \Upsilon_{u} H_{2} \\
-\Upsilon_{d} \overline{H_{2}} & \Upsilon_{d} \overline{H_{1}}
\end{array}\right),
\end{gathered}
$$

where we introduced the Higgs doublet:

$$
H \equiv\left(\begin{array}{l}
H_{1} \\
H_{2}
\end{array}\right)=\left(\begin{array}{c}
1+\phi_{1} \\
\phi_{2}
\end{array}\right) .
$$

\subsection{The spectral action for the full static SM}

We consider here the Krein-shifted operator for the static simplified model, computing the coefficients of the spectral action for its spatial part only, which is an elliptic operator. Of course, this will not give the full four-dimensional spectral action, however, we shall at least recover a part of it, valid for the spatial component of all fields under the assumption that they are time-independent. The procedure can be understood as follows. We first restrict the Krein-shifted Dirac operator, together with all its gauge fluctuations, to the 3dimensional manifold, obtaining an elliptic operator both for the leptonic and quark sectors. Then we perform the standard computation of the Gilkey-Seeley-DeWitt coefficients, using the standard formulae [20], however, we change the dimension-related constants so that they correspond to the four-dimensional case. Equivalently, this can be seen as the spectral action for the product geometry of the spatial Krein-shifted Dirac operator over a threedimensional Euclidean manifold with a circle of radius 1, for all fluctuations, which do not depend on the coordinate of the circle and resembles the dimensional reduction procedure as presented in [21].

The fluctuated Krein-shifted static Dirac operator for the Standard Model splits into the lepton and the quark sector with the lepton part,

$$
\begin{aligned}
\widetilde{D_{L}}= & i\left(\begin{array}{cc}
\mathbf{1}_{2} & \\
& -\mathbf{1}_{2}
\end{array}\right) \otimes \sigma^{j} \partial_{j}+\left(\begin{array}{cc} 
& \Phi_{l} \\
\Phi_{l}^{\dagger} &
\end{array}\right) \otimes \mathbf{1}_{2} \\
& +A_{j}\left(\begin{array}{lll}
\sigma^{3}-\mathbf{1}_{2} & \\
& & \mathbf{1}_{2}
\end{array}\right) \otimes \sigma^{j}-\left(\begin{array}{cc}
\mathbf{0}_{2} & \\
& W_{j}
\end{array}\right) \otimes \sigma^{j},
\end{aligned}
$$

where we have used the identification $M_{4}(\mathbb{C}) \otimes M_{2}(\mathbb{C}) \otimes \mathbb{C} \cong M_{4}(\mathbb{C}) \otimes M_{2}(\mathbb{C})$ and therefore omitted the third component in the expression above.

This reproduces the correct hypercharges in the leptonic sector: $0,-2,-1,-1$. We remark that for the left particles, the hypercharges are defined as coefficients in terms containing $\widetilde{\sigma}^{j}$ instead of $\sigma^{j}$. 
For the quark sector we have:

$$
\begin{aligned}
\widetilde{D_{Q}}= & i\left(\begin{array}{cc}
\mathbf{1}_{2} & \\
& -\mathbf{1}_{2}
\end{array}\right) \otimes \sigma^{j} \partial_{j} \otimes \mathbf{1}_{3}+\left(\begin{array}{cc} 
& \Phi_{q} \\
\Phi_{q}^{\dagger} &
\end{array}\right) \otimes \mathbf{1}_{2} \otimes \mathbf{1}_{3} \\
& +A_{j}\left(\begin{array}{cc}
\sigma^{3}+\frac{1}{3} \mathbf{1}_{2} & \\
& -\frac{1}{3} \mathbf{1}_{2}
\end{array}\right) \otimes \sigma^{j} \otimes \mathbf{1}_{3} \\
& -\left(\begin{array}{cc}
\mathbf{0}_{2} & \\
& W_{j}
\end{array}\right) \otimes \sigma^{j} \otimes \mathbf{1}_{3}+\left(\begin{array}{cc}
\mathbf{1}_{2} & \\
& -\mathbf{1}_{2}
\end{array}\right) \otimes \sigma^{j} \otimes G_{j} .
\end{aligned}
$$

Again, it gives correct hypercharges for quarks: $\frac{4}{3},-\frac{2}{3}, \frac{1}{3}, \frac{1}{3}$. Contributions to the action can be computed separately for the leptonic and the quark sector. The detailed computations are in the appendix A, here we present the final result in the physical parametrization.

\subsubsection{The full spectral action}

The asymptotic expansion of the spectral action for models on a four dimensional space with a fluctuated Dirac operator $D_{\omega}$ is given by

$$
\operatorname{Tr}\left(f\left(\frac{D_{\omega}}{\Lambda}\right)\right) \sim a_{4} f(0)+2 \sum_{\substack{0 \leq k<4 \\ k \text { even }}} f_{4-k} a_{k} \frac{\Lambda^{4-k}}{\Gamma\left(\frac{4-k}{2}\right)}+\mathcal{O}\left(\Lambda^{-1}\right),
$$

and reduces simply to

$$
\operatorname{Tr}\left(f\left(\frac{D_{\omega}}{\Lambda}\right)\right) \sim a_{4} f(0)+2 a_{0} f_{4} \Lambda^{4}+2 f_{2} \Lambda^{2} a_{2}+\mathcal{O}\left(\Lambda^{-1}\right),
$$

where $a_{k}$ are the so-called Gilkey-Seeley-DeWitt coefficients and can be computed explicitly - see e.g. $[6,20]$ for the detailed discussion. Here $f$ is a sufficiently regular function (see e.g. [26, chapter 2]) with $f_{k}$ being its $k$ th moment, and $\Lambda$ is the cut-off parameter.

We start with the model containing only one generation of particles. In this case we get

$$
\begin{aligned}
& a_{2}=-\frac{\kappa}{4 \pi^{2}} a \int d^{4} x|H|^{2}, \\
& a_{4}=\frac{\kappa}{8 \pi^{2}} \int d^{4} x\left[b|H|^{4}+a \operatorname{Tr}\left|D_{j} H\right|^{2}+\frac{20}{9} F^{2}+\frac{2}{3} \operatorname{Tr} W^{2}+\frac{2}{3} \operatorname{Tr} G^{2}\right],
\end{aligned}
$$

where

$$
\begin{aligned}
& a=\left|\Upsilon_{\nu}\right|^{2}+\left|\Upsilon_{e}\right|^{2}+3\left|\Upsilon_{u}\right|^{2}+3\left|\Upsilon_{d}\right|^{2}, \\
& b=\left|\Upsilon_{\nu}\right|^{4}+\left|\Upsilon_{e}\right|^{4}+3\left|\Upsilon_{u}\right|^{4}+3\left|\Upsilon_{d}\right|^{4},
\end{aligned}
$$

and $\kappa$ is the normalization of the trace.

In case of three generations we have to change the above coefficients into

$$
\begin{aligned}
& a=\operatorname{Tr}\left(\Upsilon_{\nu}^{\dagger} \Upsilon_{\nu}\right)+\operatorname{Tr}\left(\Upsilon_{e}^{\dagger} \Upsilon_{e}\right)+3 \operatorname{Tr}\left(\Upsilon_{u}^{\dagger} \Upsilon_{u}\right)+3 \operatorname{Tr}\left(\Upsilon_{d}^{\dagger} \Upsilon_{d}\right), \\
& b=\operatorname{Tr}\left(\Upsilon_{\nu}^{\dagger} \Upsilon_{\nu}\right)^{2}+\operatorname{Tr}\left(\Upsilon_{e}^{\dagger} \Upsilon_{e}\right)^{2}+3 \operatorname{Tr}\left(\Upsilon_{u}^{\dagger} \Upsilon_{u}\right)^{2}+3 \operatorname{Tr}\left(\Upsilon_{d}^{\dagger} \Upsilon_{d}\right)^{2}
\end{aligned}
$$


and we have to multiply the terms with field curvatures by a factor of 3 . As a result

$$
a_{4}=\frac{\kappa}{8 \pi^{2}} \int d^{4} x\left[b|H|^{4}+a \operatorname{Tr}\left|D_{j} H\right|^{2}+\frac{20}{3} F^{2}+2 \operatorname{Tr} W^{2}+2 \operatorname{Tr} G^{2}\right] .
$$

Taking $\kappa=4$ and ignoring the term related to the gravitational constant (i.e. the one $\sim \Lambda^{4}$ ) we end up with a model with an effective Lagrangian $\mathcal{L}=\mathcal{L}_{\text {Higgs }}+\mathcal{L}_{\text {gauge }}$, where

$$
\begin{aligned}
& \mathcal{L}_{\text {Higgs }}=\frac{b f(0)}{2 \pi^{2}}|H|^{4}-\frac{2 f_{2} \Lambda^{2} a}{\pi^{2}}|H|^{2}+\frac{a f(0)}{2 \pi^{2}} \operatorname{Tr}\left|D_{j} H\right|^{2}, \\
& \mathcal{L}_{\text {gauge }}=\frac{f(0)}{\pi^{2}}\left(\frac{10}{3} F^{2}+\operatorname{Tr} W^{2}+\operatorname{Tr} G^{2}\right) .
\end{aligned}
$$

The above result is in agreement with the one in [6, proposition 11.9], for $c=d=e=0$ in the notation used therein. Furthermore, notice also that this is consistent (up to an irrelevant global factor) with taking the static part of the Lorentzian Lagrangian for the Standard Model. Indeed, we have

$$
\begin{aligned}
-F_{\mu \nu} F^{\mu \nu}+\left|D_{\mu} H\right|^{2}-V(H) & =-2 F_{0 j} F^{0 j}-F_{j k} F^{j k}+D_{0} H^{\dagger} D_{0} H-D_{j} H^{\dagger} D_{j} H-V(H) \\
& =-F_{j k} F_{j k}-D_{j} H^{\dagger} D_{j} H-V(H)=-\left(F_{j k} F_{j k}+D_{j} H^{\dagger} D_{j} H+V(H)\right) .
\end{aligned}
$$

In particular any prediction related to the mass of the Higgs field remains unchanged.

\subsection{Wick rotated model}

Let us start with the full Krein-shifted Dirac operator in the leptonic sector,

$$
\begin{aligned}
& \widetilde{D}_{L}=i\left(\begin{array}{cc}
\mathbf{1}_{2} \otimes \sigma^{\mu} & \\
& \mathbf{1}_{2} \otimes \widetilde{\sigma}^{\mu}
\end{array}\right) \partial_{\mu}+A_{\mu}\left(\begin{array}{ll}
\left(\sigma^{3}-\mathbf{1}_{2}\right) \otimes \sigma^{\mu} & \\
& -\mathbf{1}_{2} \otimes \widetilde{\sigma}^{\mu}
\end{array}\right) \\
& +\left(\begin{array}{cc}
\mathbf{0}_{4} & \\
& W_{\mu} \otimes \widetilde{\sigma}^{\mu}
\end{array}\right)+\left(\begin{array}{cc} 
& \Phi_{l} \\
& \Phi_{l}^{\dagger}
\end{array}\right) \otimes \mathbf{1}_{2} .
\end{aligned}
$$

Its Lorentzian counterpart is of the form

$$
\begin{aligned}
& D_{L}=i\left(\mathbf{1}_{2} \otimes \sigma^{\mu} \mathbf{1}^{\mathbf{1}_{2} \otimes \tilde{\sigma}^{\mu}}\right) \partial_{\mu}+A_{\mu}\left(\left(_{\left(\sigma^{3}-\mathbf{1}_{2}\right) \otimes \sigma^{\mu}}^{-\mathbf{1}_{2} \otimes \tilde{\sigma}^{\mu}}\right)\right. \\
& +\left(\begin{array}{ll} 
& W_{\mu} \otimes \widetilde{\sigma}^{\mu} \\
\mathbf{0}_{4} &
\end{array}\right)+\left(\begin{array}{ll}
\Phi_{l}^{\dagger} & \\
& \Phi_{l}
\end{array}\right) \otimes \mathbf{1}_{2} .
\end{aligned}
$$

In what follows we perform a Wick rotation on the level of the algebra of Pauli matrices: $\sigma^{j} \rightarrow i \sigma^{j}$ and consequently $\widetilde{\sigma}^{j} \rightarrow-i \sigma^{j}$, while the $\sigma^{0}$ remains unchanged. The Wick-rotated Dirac operator in the leptonic sector is then of the form

$$
\begin{aligned}
D_{L, w}= & i\left(\begin{array}{r}
\mathbf{1}_{2}
\end{array}\right) \otimes \mathbf{1}_{2} \partial_{0}+i\left(\begin{array}{l}
\mathbf{1}_{2}-i \mathbf{1}_{2} \\
i \mathbf{1}_{2}
\end{array}\right) \otimes \sigma^{j} \partial_{j} \\
& +A_{0}\left(\begin{array}{l}
\left(\sigma^{3}-\mathbf{1}_{2}\right)^{-\mathbf{1}_{2}}
\end{array}\right) \otimes \mathbf{1}_{2}+A_{j}\left(\begin{array}{r}
i\left(\sigma^{3}-\mathbf{1}_{2}\right)^{i}
\end{array}\right) \otimes \sigma^{j} \\
& +\left(\begin{array}{c}
W_{0} \\
\mathbf{0}_{2}
\end{array}\right) \otimes \mathbf{1}_{2}-\left(\begin{array}{l}
i W_{j} \\
\mathbf{0}_{2}
\end{array}\right) \otimes \sigma^{j}+\left(\begin{array}{ll}
\Phi_{l}^{\dagger} & \\
& \Phi_{l}
\end{array}\right) \otimes \mathbf{1}_{2} .
\end{aligned}
$$


For the quark sector we have

$$
\begin{aligned}
& \widetilde{D}_{Q}=i\left(\begin{array}{rr}
\mathbf{1}_{2} \otimes \sigma^{\mu} & \\
& \mathbf{1}_{2} \otimes \widetilde{\sigma}^{\mu}
\end{array}\right) \otimes \mathbf{1}_{3} \partial_{\mu}+A_{\mu}\left(\begin{array}{rr}
\left(\sigma^{3}+\frac{1}{3} \mathbf{1}_{2}\right) \otimes \sigma^{\mu} & \\
& \frac{1}{3} \mathbf{1}_{2} \otimes \widetilde{\sigma}^{\mu}
\end{array}\right) \otimes \mathbf{1}_{3} \\
& +\left(\begin{array}{cc}
\mathbf{1}_{2} \otimes \sigma^{\mu} & \\
& \mathbf{1}_{2} \otimes \widetilde{\sigma}^{\mu}
\end{array}\right) \otimes G_{\mu}+\left(\begin{array}{cc}
\mathbf{0}_{2} & \\
& W_{\mu} \otimes \widetilde{\sigma}^{\mu}
\end{array}\right) \otimes \mathbf{1}_{3}+\left(\begin{array}{c}
\Phi_{q} \\
\Phi_{q}^{\dagger}
\end{array}\right) \otimes \mathbf{1}_{2} \otimes \mathbf{1}_{3} .
\end{aligned}
$$

Then

$$
\begin{aligned}
D_{Q}= & i\left(\mathbf{1}_{2} \otimes \sigma^{\mu} \mathbf{1}_{2} \otimes \widetilde{\sigma}^{\mu}\right) \otimes \mathbf{1}_{3} \partial_{\mu}+A_{\mu}\left(\left(\sigma^{3}+\frac{1}{3} \mathbf{1}_{2}\right) \otimes \sigma^{\mu}\right. \\
& +\left(\mathbf{1}_{2} \otimes \sigma^{\mu} \mathbf{1}_{2} \otimes \widetilde{\sigma}^{\mu}\right) \otimes G_{\mu}+\left(\begin{array}{c}
W_{\mu} \otimes \widetilde{\sigma}^{\mu} \\
\mathbf{0}_{4}
\end{array}\right) \otimes \mathbf{1}_{3}+\left(\begin{array}{cc}
\Phi_{q}^{\dagger} & \\
& \Phi_{q}
\end{array}\right) \otimes \mathbf{1}_{2} \otimes \mathbf{1}_{3},
\end{aligned}
$$

and after performing the Wick rotation we get

$$
\begin{aligned}
& D_{Q, w}=i\left(\begin{array}{c}
\mathbf{1}_{2} \\
\mathbf{1}_{2}
\end{array}\right) \otimes \mathbf{1}_{2} \otimes \mathbf{1}_{3} \partial_{0}+i\left(\begin{array}{cc} 
& -i \mathbf{1}_{2} \\
i \mathbf{1}_{2} &
\end{array}\right) \otimes \sigma^{j} \otimes \mathbf{1}_{3} \partial_{j}+\left(\begin{array}{ll}
\Phi_{q}^{\dagger} & \\
& \Phi_{q}
\end{array}\right) \otimes \mathbf{1}_{2} \otimes \mathbf{1}_{3} \\
& +A_{0}\left(\sigma^{3}+\frac{1}{3}^{\frac{1}{3} \mathbf{1}_{2}}{ }^{\mathbf{1}_{2}}\right) \otimes \mathbf{1}_{2} \otimes \mathbf{1}_{3}+i A_{j}\left(\sigma^{3}+\frac{1}{3} \mathbf{1}_{2}^{-\frac{1}{3} \mathbf{1}_{2}}\right) \otimes \sigma^{j} \otimes \mathbf{1}_{3} \\
& +\left(\begin{array}{l}
\mathbf{1}_{2} \\
\mathbf{1}_{2}
\end{array}\right) \otimes \mathbf{1}_{2} \otimes G_{0}+\left(\begin{array}{l}
\mathbf{1}_{2} \\
-\mathbf{1}_{2}
\end{array}\right) \otimes \sigma^{j} \otimes i G_{j} \\
& +\left(\begin{array}{l}
W_{0} \\
\mathbf{0}_{2}
\end{array}\right) \otimes \mathbf{1}_{2} \otimes \mathbf{1}_{3}+\left(\mathbf{0}^{-i W_{j}}\right) \otimes \sigma^{j} \otimes \mathbf{1}_{3} .
\end{aligned}
$$

Again, all further details of the computations are in the appendix B, and in what follows we present only the final expressions for the Wick-rotated model.

\subsubsection{The full spectral action}

We consider now the full model with three generations of particles. In this case, using the above results, we get

$$
a_{2}=\frac{3 \kappa}{4 \pi^{2}} a \int d^{4} x|H|^{2}
$$

and

$$
\begin{gathered}
a_{4}=\frac{\kappa}{8 \pi^{2}} \int d^{4} x\left[b|H|^{4}-a \operatorname{Tr}\left|D_{\mu} H\right|^{2}+\frac{20}{3} F^{2}+2 \operatorname{Tr}\left(W^{2}\right)+2 \operatorname{Tr}\left(G^{2}\right)\right. \\
\left.+12 \varepsilon^{j k l} F_{j k} F_{0 l}-6 \varepsilon^{j k l} \operatorname{Tr}\left(W_{j k} W_{0 l}\right)\right]
\end{gathered}
$$

where the parameters $a$ and $b$ are as before:

$$
\begin{aligned}
& a=\operatorname{Tr}\left(\Upsilon_{\nu}^{\dagger} \Upsilon_{\nu}\right)+\operatorname{Tr}\left(\Upsilon_{e}^{\dagger} \Upsilon_{e}\right)+3 \operatorname{Tr}\left(\Upsilon_{u}^{\dagger} \Upsilon_{u}\right)+3 \operatorname{Tr}\left(\Upsilon_{d}^{\dagger} \Upsilon_{d}\right), \\
& b=\operatorname{Tr}\left(\Upsilon_{\nu}^{\dagger} \Upsilon_{\nu}\right)^{2}+\operatorname{Tr}\left(\Upsilon_{e}^{\dagger} \Upsilon_{e}\right)^{2}+3 \operatorname{Tr}\left(\Upsilon_{u}^{\dagger} \Upsilon_{u}\right)^{2}+3 \operatorname{Tr}\left(\Upsilon_{d}^{\dagger} \Upsilon_{d}\right)^{2}
\end{aligned}
$$


Notice that by construction these parameters are non-negative. Taking $\kappa=4$ and considering the first terms of the asymptotic expansion (and neglecting the gravitational terms) we end up with the following Lagrangians for gauge fields and the field $H$ :

$$
\begin{aligned}
\mathcal{L}_{\text {gauge }} & =\frac{f(0)}{\pi^{2}}\left(\frac{10}{3} F^{2}+\operatorname{Tr}\left(W^{2}\right)+\operatorname{Tr}\left(G^{2}\right)+6 \varepsilon^{j k l} F_{j k} F_{0 l}-3 \varepsilon^{j k l} \operatorname{Tr}\left(W_{j k} W_{0 l}\right)\right), \\
\mathcal{L}_{H} & =\frac{b f(0)}{2 \pi^{2}}|H|^{4}+\frac{6 f_{2} \Lambda^{2}}{\pi^{2}} a|H|^{2}-\frac{a f(0)}{2 \pi^{2}} \operatorname{Tr}\left|D_{\mu} H\right|^{2} .
\end{aligned}
$$

Since the Wick rotation was performed in three spatial directions on the level of Pauli algebra, as described in the discussion preceding eq. (3.4), when going back from the Minkowski signature $(1,3)$ into the Euclidean one we have to change spatial derivatives and coordinates according to $\partial_{j} \rightarrow-i \partial_{j}$ and $A_{j} \rightarrow-i A_{j}$, respectively, and in order to preserve the spin structure we have to change the Minkowski structure constants $\varepsilon_{\mathrm{M}}^{j k l}$ into their Euclidean counterparts: $\varepsilon_{\mathrm{E}}^{j k l}=-i \varepsilon_{\mathrm{M}}^{j k l}$. As a result

$$
\begin{aligned}
-F_{\mu \nu}^{\mathrm{M}} F_{\mathrm{M}}^{\mu \nu} & =-2 F_{0 j}^{\mathrm{M}} F_{\mathrm{M}}^{0 j}-F_{j k}^{\mathrm{M}} F_{\mathrm{M}}^{j k}=2 F_{0 j}^{\mathrm{M}} F_{0 j}^{\mathrm{M}}-F_{j k}^{\mathrm{M}} F_{j k}^{\mathrm{M}} \\
& \rightarrow-2 F_{0 j}^{\mathrm{E}} F_{0 j}^{\mathrm{E}}-F_{j k}^{\mathrm{E}} F_{j k}^{\mathrm{E}}=-F_{\mu \nu}^{\mathrm{E}} F_{\mu \nu}^{\mathrm{E}},
\end{aligned}
$$

and

$$
\begin{aligned}
\left(D_{\mu} H_{\mathrm{M}}^{\dagger}\right)\left(D^{\mu} H_{\mathrm{M}}\right) & =\left(D_{0} H_{\mathrm{M}}^{\dagger}\right)\left(D_{0} H_{\mathrm{M}}\right)-\left(D_{j} H_{\mathrm{M}}^{\dagger}\right)\left(D_{j} H_{\mathrm{M}}\right) \\
& \rightarrow\left(D_{0} H_{\mathrm{E}}^{\dagger}\right)\left(D_{0} H_{\mathrm{E}}\right)+\left(D_{j} H_{\mathrm{E}}^{\dagger}\right)\left(D_{j} H_{\mathrm{E}}\right)=\left(D_{\mu} H_{\mathrm{E}}^{\dagger}\right)\left(D_{\mu} H_{\mathrm{E}}\right),
\end{aligned}
$$

so that for these terms we have

$$
-F_{\mathrm{M}}^{2}+\left|D_{\mu} H_{\mathrm{M}}\right|^{2}-V\left(H_{\mathrm{M}}\right) \rightarrow-\left(F_{\mathrm{E}}^{2}-\left|D_{\mu} H_{\mathrm{E}}\right|^{2}+V\left(H_{\mathrm{E}}\right)\right)
$$

in a complete agreement with (3.10) and (3.11). The global minus sign (together with the additional $-i$ factor from the measure) is related to the definition of an Euclidean action: $i S_{\mathrm{M}}=-S_{\mathrm{E}}$. Next, let us consider the remaining term:

$$
\varepsilon_{\mathrm{M}}^{\mu \nu \rho \sigma} F_{\mu \nu}^{\mathrm{M}} F_{\rho \sigma}^{\mathrm{M}}=4 \varepsilon_{\mathrm{M}}^{j k l} F_{0 j}^{\mathrm{M}} F_{k l}^{\mathrm{M}} \rightarrow-4 \varepsilon_{\mathrm{E}}^{j k l} F_{0 j}^{\mathrm{E}} F_{j k}^{\mathrm{E}} .
$$

Taking into account the additional global sign from the identification of $i S_{\mathrm{M}}$ with $-S_{\mathrm{E}}$, we finally see that the Lorentzian counterpart of $6 \varepsilon^{j k l} F_{j k} F_{0 l}-3 \varepsilon^{j k l} \operatorname{Tr}\left(W_{j k} W_{0 l}\right)$ is

$$
\frac{3}{2} \varepsilon^{\mu \nu \rho \sigma} F_{\mu \nu} F_{\rho \sigma}-\frac{3}{4} \varepsilon^{\mu \nu \rho \sigma} \operatorname{Tr}\left(W_{\mu \nu} W_{\rho \sigma}\right) .
$$

Therefore, the spectral action for this model contains terms that can be interpreted as the so-called $\theta$-terms in the electroweak sector [27-29]. We remark that from the above derivation of the spectral action not only the presence of such terms is deduced but also the numerical value of the electroweak vacuum angle is fixed by the model. The presence of such terms was linked with the CP-violation [28], especially for the discussion of the baryogenesis process. In contrast to the usual considerations in the physical formulation of the Standard Model, no CP-violating $\theta$-term in the QCD sector is present here. It will be interesting to see what are the physical limitations, e.g. on the energy scales on which such model is valid, from the perspective of the presence of the electroweak $\theta$-terms. The CP-violation was present in this model also on the level of algebra by the lack of the $\mathcal{J}$-symmetry [18]. 
We remark that the explicit form of the potential $V(H)$ differs from the one in the standard derivation [6], where the coefficient in the quadratic term $|H|^{2}$ contained $-2 f_{2}$ instead of $6 f_{2}$, which we have in the present model. In the usual formulation, $f$ is assumed to be, besides the others, a non-negative on the positive real half-line, so then $f_{2}$ is also nonnegative therein. If we would not allow for any relaxation of this principle, our model will not predict the Higgs mechanism, or in other words, the model could be valid only in a sector with the Higgs potential of the form $|H|^{4}+b_{1}|H|^{2}+b_{2}$ with positive $b_{1}, b_{2}$, i.e. the Higgs potential will not possess a non-trivial minimum. On the other hand, having the possibility of using function $f$ which has a negative second moment gives rise to effective action for the Standard Model with the Higgs mechanism implemented in a completely similar manner as in the usual product-like almost-commutative geometry [6]. Since all the derivations were made on the algebraic level we could, by linearity, extend the applicability of the usual methods into the case with $f$ being a difference of two positive functions. However, the discussion of the analytical aspects is required to establish the range of validity of these computational methods - see [26] for some further discussion of these aspects which are beyond the scope of this paper.

Allowing for the negative value of $f_{2}$ there is no further difference in the numerical value of the Higgs mass, which can be computed from the derived Lagrangian using the standard tools based on the renormalization group equation [6, 12]. This is because the difference in the numerical value of $f_{2}$ in the coefficient for the $|H|^{2}$ term does not affect any relation between the mass of the $W$ boson, the Higgs vacuum expectation value $v$ and the coupling constant $g_{2}$ for the $W$ boson field. Of course, the constant $f_{2}$ appears in other, purely gravitational terms, which have been deliberately neglected in these computations. Certainly, the relative sign between the cosmological term and the Einstein-Hilbert term is significant for gravity, however, this depends on another constant $f_{4}$, which contributes to the factor in front of the cosmological term. In the usual Einstein-Hilbert action the signs of these two terms are opposite, which is consistent with our results provided that $f_{4}$ is positive. The only potential problem for the Euclidean action is its overall positivity, yet this may depend on the overall sign, which depends on the Wick-rotation scheme. We hope that the question of relative signs and spectral action expansion coefficients for the full Lorentzian model will be effectively tackled by extending the results of [25] to Dirac-type operators.

\section{Conclusions and outlook}

The presented noncommutative geometric model describing the particle interaction appears to be the closest to the observed Standard Model. The derived bosonic spectral action gives all correct terms and, in addition, new, topological $\theta$-terms. While the latter has no significance for the dynamics of the model, it may play a role in the quantum effects for the electroweak sector. These terms are, in principle, not excluded and have been discussed in literature [27-29]. The result signifies also that computing the spectral action for the Wickrotated Lorentzian Dirac operator is important. Possibly, the next step shall be to compute the genuine Lorentzian spectral action using the tools that are at present available for the 
Laplace-type operators [25]. Furthermore, possible relations of non-product geometries with bundle-like structures over noncommutative manifolds [30] as well to the inclusion of gravity for this non-product geometry (see [31] for a link between nonproduct geometries and gravity) shall also be explored and examined. Finally, it shall be interesting to see possible extensions of the model, both in the direction of scalar conformal modifications that can help to fix the Higgs mass as well as extensions of the Pati-Salam type [32, 33].

\section{A The static spectral action}

\section{A.1 Leptonic sector}

In the leptonic sector we have

$$
{\widetilde{D_{L}}}^{2}=-\left(\mathbf{1}_{4} \otimes \mathbf{1}_{2}\right) \Delta-a^{j} \partial_{j}-b,
$$

where,

$$
\begin{aligned}
& a^{j}=-2 i\left(A_{j}\left(\begin{array}{lll}
\sigma^{3}-\mathbf{1}_{2} & \\
& & -\mathbf{1}_{2}
\end{array}\right)+\left(\begin{array}{ll}
\mathbf{0}_{2} & \\
& W_{j}
\end{array}\right)\right) \otimes \mathbf{1}_{2},
\end{aligned}
$$

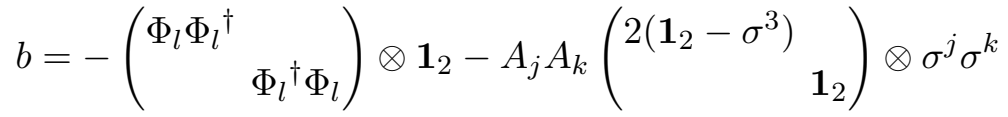

$$
\begin{aligned}
& -\left(\begin{array}{cc}
\mathbf{0}_{2} & \\
& W_{j} W_{k}
\end{array}\right) \otimes \sigma^{j} \sigma^{k}+\left(\begin{array}{cc}
W_{j} \Phi_{l}^{\dagger} & \\
& \Phi_{l} W_{j}
\end{array}\right) \otimes \sigma^{j}+2\left(\begin{array}{cc}
\mathbf{0}_{2} & \\
& W_{j}
\end{array}\right) A_{j} \otimes \mathbf{1}_{2} \\
& -i\left({ }_{-\partial_{j} \Phi_{l}^{\dagger}}{ }^{\partial_{j} \Phi_{l}}\right) \otimes \sigma^{j}-i\left(\begin{array}{ll}
\mathbf{0}_{2} & \\
& \partial_{j} W_{k}
\end{array}\right) \otimes \sigma^{j} \sigma^{k}
\end{aligned}
$$

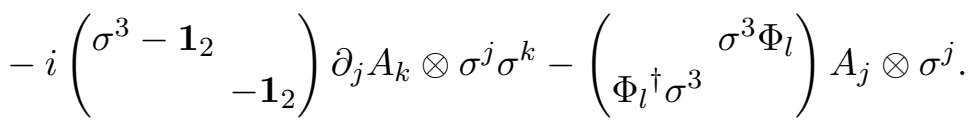

As a result, following the notation of [20], we have $\omega_{j}=\frac{1}{2} a^{j}$, so that

$$
\begin{aligned}
\Omega_{i j} & =\partial_{i} \omega_{j}-\partial_{j} \omega_{i}+\omega_{i} \omega_{j}-\omega_{j} \omega_{i} \\
& =-i F_{i j}\left(\begin{array}{cc}
\sigma^{3}-\mathbf{1}_{2} & \\
& \\
& -\mathbf{1}_{2}
\end{array}\right) \otimes \mathbf{1}_{2}-i\left(\begin{array}{cc}
\mathbf{0}_{2} & \\
& W_{i j}
\end{array}\right) \otimes \mathbf{1}_{2}
\end{aligned}
$$

with

$$
F_{i j}=\partial_{i} A_{j}-\partial_{j} A_{i}, \quad W_{i j}=\partial_{i} W_{j}-\partial_{j} W_{i}-i\left[W_{i}, W_{j}\right] .
$$

Next we compute,

$$
\begin{aligned}
& E=b-\partial_{j} \omega_{j}-\omega_{j} \omega_{j}
\end{aligned}
$$

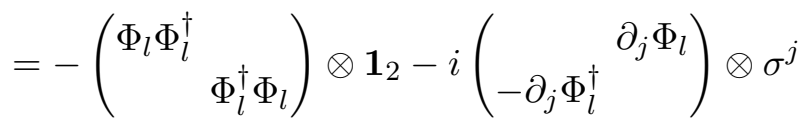

$$
\begin{aligned}
& +\frac{1}{2}\left(\begin{array}{ll}
\mathbf{0}_{2} & \\
& W_{j k}
\end{array}\right) \otimes \varepsilon^{j k l} \sigma^{l}+\frac{1}{2} F_{j k}\left(\begin{array}{lll}
\sigma^{3}-\mathbf{1}_{2} & \\
& & \\
& & -\mathbf{1}_{2}
\end{array}\right) \otimes \varepsilon^{j k l} \sigma^{l} \\
& -A_{j}\left(\Phi_{l}^{\dagger} \sigma^{3} \sigma^{3} \Phi_{l}\right) \otimes \sigma^{j}+\left(W_{j} \Phi_{l}^{\dagger} \Phi_{l} W_{j}\right) \otimes \sigma^{j} .
\end{aligned}
$$


We get then

$$
\operatorname{Tr}(E)=-4 \kappa \operatorname{Tr}\left(\Phi_{l}^{\dagger} \Phi_{l}\right)
$$

and furthermore,

$$
\operatorname{Tr}\left(\Omega_{i j} \Omega_{i j}\right)=-2 \kappa\left(6 F^{2}+\operatorname{Tr}\left(W^{2}\right)\right),
$$

where $\kappa$ is the normalization of the trace (i.e. everything within the bracket is computed for the unnormalized trace) and $W^{2}=W_{j k} W_{j k}$. Moreover,

$$
\begin{aligned}
\kappa^{-1} \operatorname{Tr}\left(E^{2}\right)= & 4 \operatorname{Tr}\left(\Phi_{l}^{\dagger} \Phi_{l}\right)^{2}+4 \operatorname{Tr}\left[\left(\partial_{j} \Phi_{l}^{\dagger}\right)\left(\partial_{j} \Phi_{l}\right)\right]+4 A^{2} \operatorname{Tr}\left(\Phi_{l}^{\dagger} \Phi_{l}\right)+4 \operatorname{Tr}\left(W_{j} W_{j} \Phi_{l}^{\dagger} \Phi_{l}\right) \\
& +4 i A_{j} \operatorname{Tr}\left[\left(\left(\partial_{j} \Phi_{l}\right) \Phi_{l}^{\dagger}-\Phi_{l}\left(\partial_{j} \Phi_{l}^{\dagger}\right)\right) \sigma^{3}\right]-4 i \operatorname{Tr}\left[\left(\Phi_{l}^{\dagger}\left(\partial_{j} \Phi_{l}\right)-\left(\partial_{j} \Phi_{l}^{\dagger}\right) \Phi_{l}\right) W_{j}\right] \\
& -8 A_{j} \operatorname{Tr}\left(\Phi_{l}^{\dagger} \sigma^{3} \Phi_{l} W_{j}\right)+6 F^{2}+\operatorname{Tr}\left(W^{2}\right) .
\end{aligned}
$$

As a result, in the leptonic sector we have

$$
\begin{aligned}
a_{2}= & \frac{1}{(4 \pi)^{2}} \int d^{4} x \operatorname{Tr} E=-\frac{\kappa}{4 \pi^{2}} \int d^{4} x \operatorname{Tr}\left(\Phi_{l}^{\dagger} \Phi_{l}\right), \\
a_{4}= & \frac{1}{16 \pi^{2}} \frac{1}{12} \int d^{4} x\left(6 \operatorname{Tr}\left(E^{2}\right)+\operatorname{Tr}\left(\Omega_{i j} \Omega_{i j}\right)\right) \\
= & \frac{\kappa}{48 \pi^{2}} \int d^{4} x\left[6 \left(\operatorname{Tr}\left(\Phi_{l}^{\dagger} \Phi_{l}\right)^{2}+\operatorname{Tr}\left[\left(\partial_{j} \Phi_{l}^{\dagger}\right)\left(\partial_{j} \Phi_{l}\right)\right]+A^{2} \operatorname{Tr}\left(\Phi_{l}^{\dagger} \Phi_{l}\right)\right.\right. \\
& \quad+\operatorname{Tr}\left(W_{j} W_{j} \Phi_{l}^{\dagger} \Phi_{l}\right)+i A_{j} \operatorname{Tr}\left[\left(\left(\partial_{j} \Phi_{l}\right) \Phi_{l}^{\dagger}-\Phi_{l}\left(\partial_{j} \Phi_{l}^{\dagger}\right)\right) \sigma^{3}\right] \\
& \left.\quad-i \operatorname{Tr}\left[\left(\Phi_{l}^{\dagger}\left(\partial_{j} \Phi_{l}\right)-\left(\partial_{j} \Phi_{l}^{\dagger}\right) \Phi_{l}\right) W_{j}\right]-2 A_{j} \operatorname{Tr}\left(\Phi_{l}^{\dagger} \sigma^{3} \Phi_{l} W_{j}\right)\right) \\
& \left.+6 F^{2}+\operatorname{Tr}\left(W^{2}\right)\right] .
\end{aligned}
$$

Using the parametrization from the section 3.1 in this sector we then have

$$
\Phi_{l}^{\dagger} \Phi_{l}=\left(\begin{array}{cc}
\left|\Upsilon_{\nu}\right|^{2}\left|H_{1}\right|^{2}+\left|\Upsilon_{e}\right|^{2}\left|H_{2}\right|^{2} & \left|\Upsilon_{\nu}\right|^{2} \overline{H_{1}} H_{2}-\left|\Upsilon_{e}\right|^{2} H_{2} \overline{H_{1}} \\
\left|\Upsilon_{\nu}\right|^{2} \overline{H_{2}} H_{1}-\left|\Upsilon_{e}\right|^{2} H_{1} \overline{H_{2}} & \left|\Upsilon_{\nu}\right|^{2}\left|H_{2}\right|^{2}+\left|\Upsilon_{e}\right|^{2}\left|H_{1}\right|^{2}
\end{array}\right),
$$

and as a result

$$
a_{2}=-\frac{\kappa}{4 \pi^{2}}\left(\left|\Upsilon_{e}\right|^{2}+\left|\Upsilon_{\nu}\right|^{2}\right) \int d^{4} x|H|^{2}
$$

Furthermore we have

$$
\begin{aligned}
\operatorname{Tr}\left(\Phi_{l}^{\dagger} \Phi_{l}\right)^{2} & =\left(\left|\Upsilon_{\nu}\right|^{4}+\left|\Upsilon_{e}\right|^{4}\right)|H|^{4}, \\
\operatorname{Tr}\left[\left(\partial_{j} \Phi_{l}^{\dagger}\right)\left(\partial_{j} \Phi_{l}\right)\right] & =\left(\left|\Upsilon_{\nu}\right|^{2}+\left|\Upsilon_{e}\right|^{2}\right)\left|\partial_{j} H\right|^{2}, \\
\left(A_{j} A_{j}\right) \operatorname{Tr}\left(\Phi_{l}^{\dagger} \Phi_{l}\right) & =\left(A_{j} A_{j}\right)\left(\left|\Upsilon_{\nu}\right|^{2}+\left|\Upsilon_{e}\right|^{2}\right)|H|^{2} .
\end{aligned}
$$

Next, we decompose the $W$ field in terms of Pauli matrices,

$$
W_{j}=W_{j, 1} \sigma^{1}+W_{j, 2} \sigma^{2}+W_{j, 3} \sigma^{3},
$$

so that

$$
\operatorname{Tr}\left(W_{j} W_{j} \Phi_{l}^{\dagger} \Phi_{l}\right)=\left(W_{j} W_{j}\right)\left(\left|\Upsilon_{\nu}\right|^{2}+\left|\Upsilon_{e}\right|^{2}\right)|H|^{2}
$$


Next, we compute

$$
\begin{gathered}
i A_{j} \operatorname{Tr}\left[\left(\left(\partial_{j} \Phi_{l}\right) \Phi_{l}^{\dagger}-\Phi_{l}\left(\partial_{j} \Phi_{l}^{\dagger}\right)\right) \sigma^{3}\right]=i A_{j}\left(\left|\Upsilon_{\nu}\right|^{2}+\left|\Upsilon_{e}\right|^{2}\right)\left(H^{\dagger} \partial_{j} H-\partial_{j} H^{\dagger} H\right), \\
-i \operatorname{Tr}\left[\left(\Phi_{l}^{\dagger}\left(\partial_{j} \Phi_{l}\right)-\left(\partial_{j} \Phi_{l}^{\dagger}\right) \Phi_{l}\right) W_{j}\right]=-i\left(\left|\Upsilon_{\nu}\right|^{2}+\left|\Upsilon_{e}\right|^{2}\right)\left[W_{j, 3}\left(\overline{H_{1}} \partial_{j} H_{1}-H_{1} \partial_{j} \overline{H_{1}}-\overline{H_{2}} \partial_{j} H_{2}+H_{2} \partial_{j} \overline{H_{2}}\right)\right. \\
+\left(W_{j, 1}-i W_{j, 2}\right)\left(\overline{H_{2}} \partial_{j} H_{1}-H_{1} \partial_{j} \overline{H_{2}}\right) \\
\left.+\left(W_{j, 1}+i W_{j, 2}\right)\left(\overline{H_{1}} \partial_{j} H_{2}-H_{2} \partial_{j} \overline{H_{1}}\right)\right] \\
-2 A_{j} \operatorname{Tr}\left[\Phi_{l}^{\dagger} \sigma^{3} \Phi_{l} W_{j}\right]=-2 A_{j}\left(\left|\Upsilon_{\nu}\right|^{2}+\left|\Upsilon_{e}\right|^{2}\right)\left[\left(W_{j, 1}-i W_{j, 2}\right) H_{1} \overline{H_{2}}\right. \\
\left.+\left(W_{j, 1}+i W_{j, 2}\right) \overline{H_{1}} H_{2}+W_{j, 3}\left(\left|H_{1}\right|^{2}-\left|H_{2}\right|^{2}\right)\right] .
\end{gathered}
$$

Let us now verify whether these terms can be written using the covariant derivative of the Higgs doublet,

$$
D_{j} H=\partial_{j} H+i W_{j} H-i A_{j} H
$$

We check,

$$
\begin{aligned}
\operatorname{Tr}\left|D_{j} H\right|^{2}= & \operatorname{Tr}\left[\left|\partial_{j} H\right|^{2}+i\left(\partial_{j} H^{\dagger} W_{j} H-H^{\dagger} W_{j} \partial_{j} H\right)\right. \\
& \left.+i A_{j}\left(H^{\dagger} \partial_{j} H-\partial_{j} H^{\dagger} H\right)-2 A_{j} H^{\dagger} W_{j} H+W_{j} W_{j}|H|^{2}+A^{2}|H|^{2}\right] .
\end{aligned}
$$

The only terms that are potentially different that the ones in the coefficient $a_{4}$ are

$$
2 A_{j} \operatorname{Tr}\left(H^{\dagger} W_{j} H\right), \quad i \operatorname{Tr}\left(\partial_{j} H^{\dagger} W_{j} H-H^{\dagger} W_{j} \partial_{j} H\right)
$$

but simple calculation shows that

$$
\begin{aligned}
A_{j} \operatorname{Tr}\left(H^{\dagger} W_{j} H\right)= & A_{j}\left[W_{j, 1}\left(\overline{H_{1}} H_{2}-\overline{H_{2}} H_{1}\right)\right. \\
& \left.+i W_{j, 2}\left(\overline{H_{1}} H_{2}-\overline{H_{2}} H_{1}\right)+W_{j, 3}\left(\left|H_{1}\right|^{2}-\left|H_{2}\right|^{2}\right)\right]
\end{aligned}
$$

and

$$
\begin{aligned}
\operatorname{Tr}\left(\partial_{j} H^{\dagger} W_{j} H-H^{\dagger} W_{j} \partial_{j} H\right)= & W_{j, 1}\left(\partial_{j} \overline{H_{1}} H_{2}+\partial_{j} \overline{H_{2}} H_{1}-\overline{H_{1}} \partial_{j} H_{2}-\overline{H_{2}} \partial_{j} H_{1}\right) \\
& +W_{j, 2}\left(\partial_{j} \overline{H_{1}} H_{2}-\partial_{j} \overline{H_{2}} H_{1}-\overline{H_{1}} \partial_{j} H_{2}+\overline{H_{2}} \partial_{j} H_{1}\right) \\
& +W_{j, 3}\left(\partial_{j} \overline{H_{1}} H_{1}-\partial_{j} \overline{H_{2}} H_{2}-\overline{H_{1}} \partial_{j} H_{1}+\overline{H_{2}} \partial_{j} H_{2}\right)
\end{aligned}
$$

in a complete agreement with $a_{4}$.

Therefore,

$$
a_{4}=\frac{\kappa}{8 \pi^{2}} \int d^{4} x\left[\left(\left|\Upsilon_{\nu}\right|^{4}+\left|\Upsilon_{e}\right|^{4}\right)|H|^{4}+\left(\left|\Upsilon_{\nu}\right|^{2}+\left|\Upsilon_{e}\right|^{2}\right) \operatorname{Tr}\left|D_{j} H\right|^{2}+F^{2}+\frac{1}{6} \operatorname{Tr} W^{2}\right]
$$

\section{A.2 Quark sector}

In this sector we have

$$
\widetilde{D}_{Q}^{2}=-\left(\mathbf{1}_{4} \otimes \mathbf{1}_{2} \otimes \mathbf{1}_{3}\right) \Delta-a^{j} \partial_{j}-b,
$$


where

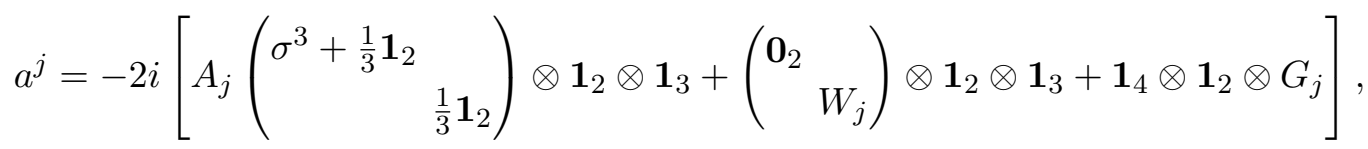

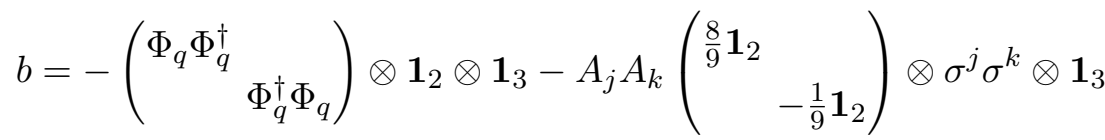

$$
\begin{aligned}
& -\left(\begin{array}{ll}
\mathbf{0}_{2} & \\
& \\
& W_{j} W_{k}
\end{array}\right) \otimes \sigma^{j} \sigma^{k} \otimes \mathbf{1}_{3}-\mathbf{1}_{4} \otimes \sigma^{j} \sigma^{k} \otimes G_{j} G_{k} \\
& -i\left(\begin{array}{ll}
\partial_{j} \Phi_{q}^{\dagger} & \partial_{j} \Phi_{q}
\end{array}\right) \otimes \sigma^{j} \otimes \mathbf{1}_{3}-i\left(\begin{array}{ll}
\mathbf{0}_{2} & \\
& \partial_{j} W_{k}
\end{array}\right) \otimes \sigma^{j} \sigma^{k} \otimes \mathbf{1}_{3} \\
& -i 1_{4} \otimes \sigma^{j} \sigma^{k} \otimes \partial_{j} G_{k}-A_{j}\left({ }_{\Phi_{q}^{\dagger} \sigma^{3}} \sigma^{3} \Phi_{q}\right) \otimes \sigma^{j} \otimes \mathbf{1}_{3} \\
& +\left(W_{j} \Phi_{q}^{\dagger} \Phi_{q} W_{j}\right) \otimes \sigma^{j} \otimes \mathbf{1}_{3}-i\left(\partial_{j} A_{k}\right)\left(\begin{array}{ll}
\sigma^{3}+\frac{1}{2} \mathbf{1}_{2} & \\
& \frac{1}{3} \mathbf{1}_{2}
\end{array}\right) \otimes \sigma^{j} \sigma^{k} \otimes \mathbf{1}_{3}
\end{aligned}
$$

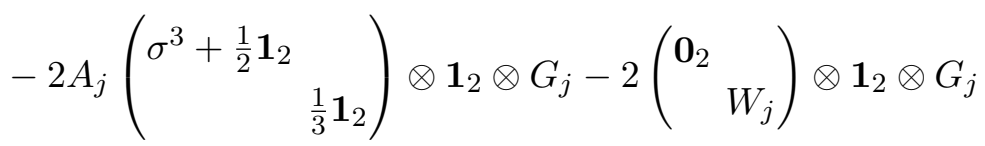

$$
\begin{aligned}
& -\frac{2}{3} A_{j} A_{k}\left(\begin{array}{cc}
\sigma^{3} & \\
& \mathbf{0}_{2}
\end{array}\right) \otimes \sigma^{j} \sigma^{k} \otimes \mathbf{1}_{3}-\frac{2}{3}\left(\begin{array}{cc}
\mathbf{0}_{2} & \\
& A_{j} W_{j}
\end{array}\right) \otimes \mathbf{1}_{2} \otimes \mathbf{1}_{3} .
\end{aligned}
$$

Therefore,

$$
\begin{aligned}
E= & b-\partial_{j} \omega_{j}-\omega_{j} \omega_{j} \\
= & -\left(\begin{array}{cc}
\Phi_{q} \Phi_{q}^{\dagger} & \\
& \Phi_{q}^{\dagger} \Phi_{q}
\end{array}\right) \otimes \mathbf{1}_{2} \otimes \mathbf{1}_{3}-i\left(\begin{array}{cc}
\partial_{j} \Phi_{q} \\
-\partial_{j} \Phi_{q}^{\dagger}
\end{array}\right) \otimes \sigma^{j} \otimes \mathbf{1}_{3} \\
& +\frac{1}{2} F_{j k}\left(\begin{array}{cc}
\sigma^{3} & \\
& \mathbf{0}_{2}
\end{array}\right) \otimes \varepsilon^{j k l} \sigma^{l} \otimes \mathbf{1}_{3}+\frac{1}{2}\left(\begin{array}{cc}
\mathbf{0}_{2} & \\
& W_{j k}
\end{array}\right) \otimes \varepsilon^{j k l} \sigma^{l} \otimes \mathbf{1}_{3}+\frac{1}{2} \mathbf{1}_{4} \otimes \varepsilon^{j k l} \sigma^{l} \otimes G_{j k} \\
& -A_{j}\left(\begin{array}{cc}
\sigma^{3} \Phi_{q} \\
\Phi_{q}^{\dagger} \sigma^{3}
\end{array}\right) \otimes \sigma^{j} \otimes \mathbf{1}_{3}+\left(\begin{array}{cc}
W_{j} \Phi_{q}^{\dagger} & \Phi_{q} W_{j}
\end{array}\right) \otimes \sigma^{j} \otimes \mathbf{1}_{3} \\
& +\frac{1}{6} F_{j k} \mathbf{1}_{4} \otimes \varepsilon^{j k l} \sigma^{l} \otimes \mathbf{1}_{3},
\end{aligned}
$$

where again

$$
G_{i j}=\partial_{i} G_{j}-\partial_{j} G_{i}-i\left[G_{i}, G_{j}\right]
$$

As a result,

$$
\kappa^{-1} \operatorname{Tr}(E)=-12 \operatorname{Tr}\left(\Phi_{q}^{\dagger} \Phi_{q}\right)
$$

and

$$
\kappa^{-1} \operatorname{Tr}\left(\Omega_{i j} \Omega_{i j}\right)=-2\left(\frac{22}{3} F^{2}+3 \operatorname{Tr}\left(W^{2}\right)+4 \operatorname{Tr}\left(G^{2}\right)\right),
$$


where we use short notation $G^{2}=G_{i j} G_{i j}$, and the full second contribution reads,

$$
\begin{aligned}
\kappa^{-1} \operatorname{Tr}\left(E^{2}\right)= & 12 \operatorname{Tr}\left(\Phi_{q}^{\dagger} \Phi_{q}\right)^{2}+12 \operatorname{Tr}\left[\left(\partial_{j} \Phi_{q}^{\dagger}\right)\left(\partial_{j} \Phi_{q}\right)\right]+12 A^{2} \operatorname{Tr}\left(\Phi_{q}^{\dagger} \Phi_{q}\right) \\
& +12 \operatorname{Tr}\left(W_{j} W_{j} \Phi_{q}^{\dagger} \Phi_{q}\right)+12 i A_{j} \operatorname{Tr}\left[\left(\left(\partial_{j} \Phi_{q}\right) \Phi_{q}^{\dagger}-\Phi_{q}\left(\partial_{j} \Phi_{q}^{\dagger}\right)\right) \sigma^{3}\right] \\
& -12 i \operatorname{Tr}\left[\left(\Phi_{q}^{\dagger}\left(\partial_{j} \Phi_{q}\right)-\left(\partial_{j} \Phi_{q}^{\dagger}\right) \Phi_{q}\right) W_{j}\right]-24 A_{j} \operatorname{Tr}\left(\Phi_{q}^{\dagger} \sigma^{3} \Phi_{q} W_{j}\right) \\
& +\frac{22}{3} F^{2}+3 \operatorname{Tr}\left(W^{2}\right)+4 \operatorname{Tr}\left(G^{2}\right) .
\end{aligned}
$$

As a result, in the quark sector we have

$$
\begin{aligned}
a_{2}= & -\frac{\kappa}{4 \pi^{2}} \int d^{4} x 3 \operatorname{Tr}\left(\Phi_{q}^{\dagger} \Phi_{q}\right), \\
a_{4}= & \frac{\kappa}{48 \pi^{2}} \int d^{4} x\left[1 8 \left(\operatorname{Tr}\left(\Phi_{q}^{\dagger} \Phi_{q}\right)^{2}+\operatorname{Tr}\left[\left(\partial_{j} \Phi_{q}^{\dagger}\right)\left(\partial_{j} \Phi_{q}\right)\right]+A^{2} \operatorname{Tr}\left(\Phi_{q}^{\dagger} \Phi_{q}\right)\right.\right. \\
& +\operatorname{Tr}\left(W_{j} W_{j} \Phi_{q}^{\dagger} \Phi_{q}\right)+i A_{j} \operatorname{Tr}\left[\left(\left(\partial_{j} \Phi_{q}\right) \Phi_{q}^{\dagger}-\Phi_{q}\left(\partial_{j} \Phi_{q}^{\dagger}\right)\right) \sigma^{3}\right] \\
& \left.-i \operatorname{Tr}\left[\left(\Phi_{q}^{\dagger}\left(\partial_{j} \Phi_{q}\right)-\left(\partial_{j} \Phi_{q}^{\dagger}\right) \Phi_{q}\right) W_{j}\right]-2 A_{j} \operatorname{Tr}\left(\Phi_{q}^{\dagger} \sigma^{3} \Phi_{q} W_{j}\right)\right) . \\
& \left.+\frac{22}{3} F^{2}+3 \operatorname{Tr}\left(W^{2}\right)+4 \operatorname{Tr}\left(G^{2}\right)\right] .
\end{aligned}
$$

In a completely similar manner as for the leptonic sector we derive:

$$
a_{2}=-\frac{\kappa}{4 \pi^{2}}\left(3\left|\Upsilon_{u}\right|^{2}+3\left|\Upsilon_{d}\right|^{2}\right) \int d^{4} x|H|^{2}
$$

and

$$
\begin{aligned}
a_{4}=\frac{\kappa}{8 \pi^{2}} \int d^{4} x & {\left[\left(3\left|\Upsilon_{u}\right|^{4}+3\left|\Upsilon_{d}\right|^{4}\right)|H|^{4}+\left(3\left|\Upsilon_{u}\right|^{2}+3\left|\Upsilon_{d}\right|^{2}\right) \operatorname{Tr}\left|D_{j} H\right|^{2}\right.} \\
+ & \left.\frac{11}{9} F^{2}+\frac{1}{2} \operatorname{Tr} W^{2}+\frac{2}{3} \operatorname{Tr} G^{2}\right] .
\end{aligned}
$$

\section{B The Wick rotated model}

\section{B.1 Leptonic sector}

Starting with the Wick rotated Dirac operator (3.4) we get

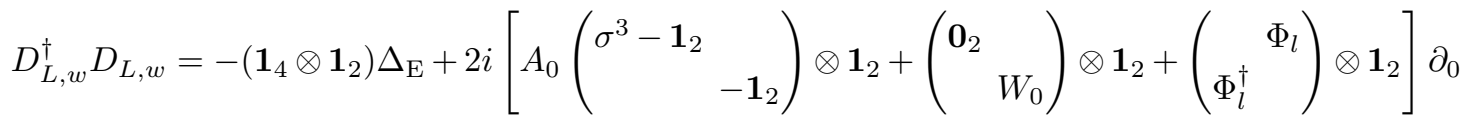

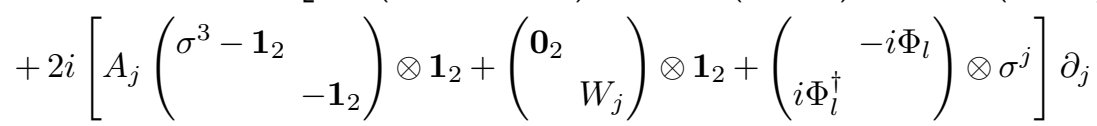

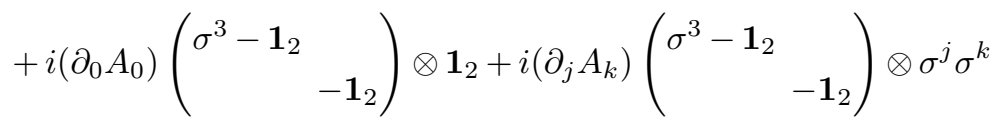

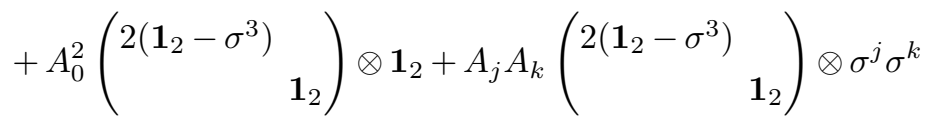




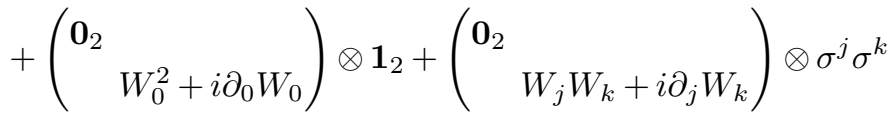

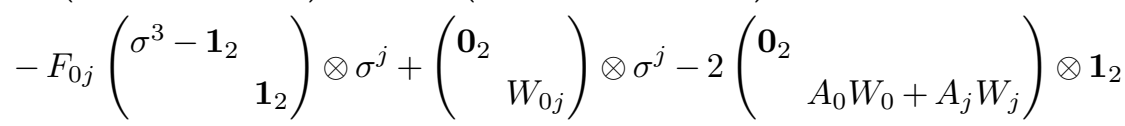

$$
\begin{aligned}
& +i\left(\begin{array}{cc} 
& \partial_{0} \Phi_{l} \\
\partial_{0} \Phi_{l}^{\dagger} &
\end{array}\right) \otimes \mathbf{1}_{2}+i\left(\begin{array}{ll} 
& -i \partial_{j} \Phi_{l} \\
i \partial_{j} \Phi_{l}^{\dagger} &
\end{array}\right) \otimes \sigma^{j}+\left(\begin{array}{cc}
\Phi_{l} \Phi_{l}^{\dagger} & \\
& \Phi_{l}^{\dagger} \Phi_{l}
\end{array}\right) \otimes \mathbf{1}_{2} \\
& +A_{0}\left(\Phi_{l}^{\dagger}\left(\sigma^{3}-2 \cdot \mathbf{1}_{2}\right)^{\left(\sigma^{3}-2 \cdot \mathbf{1}_{2}\right) \Phi_{l}}\right) \otimes \mathbf{1}_{2}+\left(W_{0} \Phi_{l}^{\dagger} \Phi_{l} W_{0}\right) \otimes \mathbf{1}_{2} \\
& +A_{j}\left(i \Phi_{l}^{\dagger}\left(\sigma^{3}-2 \cdot \mathbf{1}_{2}\right)^{-i\left(\sigma^{3}-2 \cdot \mathbf{1}_{2}\right) \Phi_{l}}\right) \otimes \sigma^{j}+\left({i W_{j} \Phi_{l}^{\dagger}}^{-i \Phi_{l} W_{j}}\right) \otimes \sigma^{j} .
\end{aligned}
$$

Writing $D_{L, w}^{\dagger} D_{L, w}$ in the canonical form $-\left(\mathbf{1}_{4} \otimes \mathbf{1}_{2}\right) \Delta_{\mathrm{E}}-2 \omega_{\mu} \partial_{\mu}-b$ (with the Euclidean summation) we get

$$
\begin{aligned}
& E=\frac{1}{2} F_{j k} \varepsilon^{j k l}\left(\begin{array}{ccc}
\sigma^{3}-\mathbf{1}_{2} & \\
& & -\mathbf{1}_{2}
\end{array}\right) \otimes \sigma^{l}+F_{0 j}\left(\begin{array}{lll}
\sigma^{3}-\mathbf{1}_{2} & \\
& & \mathbf{1}_{2}
\end{array}\right) \otimes \sigma^{j}
\end{aligned}
$$

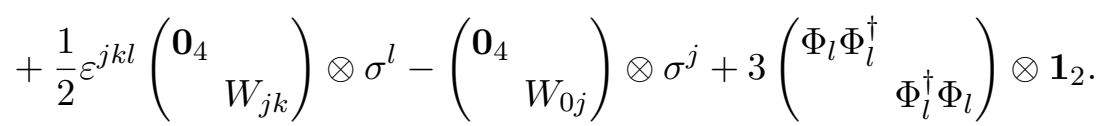

Its trace is therefore

$$
\operatorname{Tr}(E)=12 \kappa \operatorname{Tr}\left(\Phi_{l}^{\dagger} \Phi_{l}^{\dagger}\right)
$$

Furthermore, we have

$$
\kappa^{-1} \operatorname{Tr}\left(E^{2}\right)=6 F^{2}+\operatorname{Tr}\left(W^{2}\right)+36 \operatorname{Tr}\left(\Phi_{l}^{\dagger} \Phi_{l}\right)^{2}+4 \varepsilon^{j k l} F_{j k} F_{0 l}-2 \varepsilon^{j k l} \operatorname{Tr}\left(W_{j k} W_{0 l}\right),
$$

where now $F^{2}=F_{\mu \nu} F_{\mu \nu}=F_{j k} F_{j k}+2 F_{0 j} F_{0 j}$ and similarly for $W^{2}$.

Next, we have

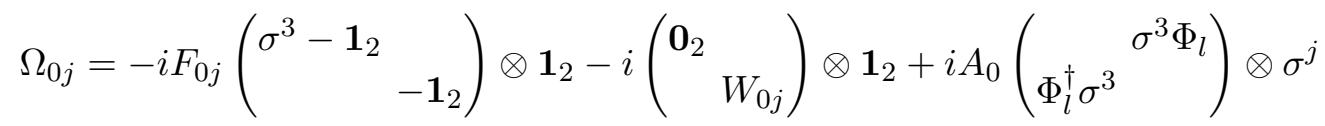

$$
\begin{aligned}
& -i\left(W_{0} \Phi_{l}^{\dagger} \Phi_{l} W_{0}\right) \otimes \sigma^{j}-A_{j}\left(\Phi_{l}^{\dagger} \sigma^{3}-\sigma^{3} \Phi_{l}\right) \otimes \mathbf{1}_{2}+\left(W_{j} \Phi_{l}^{\dagger}-\Phi_{l} W_{j}\right) \otimes \mathbf{1}_{2} \\
& -2 i\left(\begin{array}{cc}
\Phi_{l} \Phi_{l}^{\dagger} & \\
& -\Phi_{l}^{\dagger} \Phi_{l}
\end{array}\right) \otimes \sigma^{j}+i\left(\partial_{j} \Phi_{l}^{\dagger} \partial_{j} \Phi_{l}\right) \otimes \mathbf{1}_{2}+\left(\partial_{0} \Phi_{l}^{\dagger}-\partial_{0} \Phi_{l}\right) \otimes \sigma^{j},
\end{aligned}
$$

hence

$$
\begin{aligned}
\kappa^{-1} \operatorname{Tr}\left(\Omega_{0 j} \Omega_{0 j}\right)= & -12 F_{0 j} F_{0 j}-2 \operatorname{Tr}\left(W_{0 j} W_{0 j}\right)-12 A_{0}^{2} \operatorname{Tr}\left(\Phi_{l}^{\dagger} \Phi_{l}\right)-12 \operatorname{Tr}\left(W_{0}^{2} \Phi_{l}^{\dagger} \Phi_{l}\right) \\
& -4 A_{j}^{2} \operatorname{Tr}\left(\Phi_{l}^{\dagger} \Phi_{l}\right)-4 \operatorname{Tr}\left(W_{j}^{2} \Phi_{l}^{\dagger} \Phi_{l}\right)-48 \operatorname{Tr}\left(\Phi_{l}^{\dagger} \Phi_{l}\right)^{2}+24 A_{0} \operatorname{Tr}\left(\Phi_{l}^{\dagger} \sigma^{3} \Phi_{l} W_{0}\right) \\
& +8 A_{j} \operatorname{Tr}\left(\Phi_{l}^{\dagger} \sigma^{3} \Phi_{l} W_{j}\right)-4 \operatorname{Tr}\left[\left(\partial_{j} \Phi_{l}\right)^{\dagger}\left(\partial_{j} \Phi_{l}\right)\right]-12 \operatorname{Tr}\left[\left(\partial_{0} \Phi_{l}\right)^{\dagger}\left(\partial_{0} \Phi_{l}\right)\right] \\
& -12 i A_{0} \operatorname{Tr}\left[\left(\left(\partial_{0} \Phi_{l}\right) \Phi_{l}^{\dagger}-\Phi_{l}\left(\partial_{0} \Phi_{l}^{\dagger}\right)\right) \sigma^{3}\right]-4 i A_{j} \operatorname{Tr}\left[\left(\left(\partial_{j} \Phi_{l}\right) \Phi_{l}^{\dagger}-\Phi_{l}\left(\partial_{j} \Phi_{l}^{\dagger}\right)\right) \sigma^{3}\right] \\
& +12 i \operatorname{Tr}\left[\left(\Phi_{l}^{\dagger}\left(\partial_{0} \Phi_{l}\right)-\left(\partial_{0} \Phi_{l}^{\dagger}\right) \Phi_{l}\right) W_{0}\right]+4 i \operatorname{Tr}\left[\left(\Phi_{l}^{\dagger}\left(\partial_{j} \Phi_{l}\right)-\left(\partial_{j} \Phi_{l}^{\dagger}\right) \Phi_{l}\right) W_{j}\right] .
\end{aligned}
$$


Moreover,

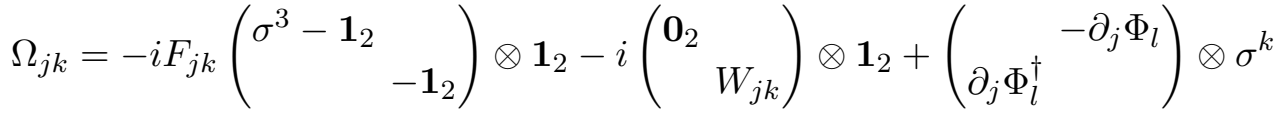

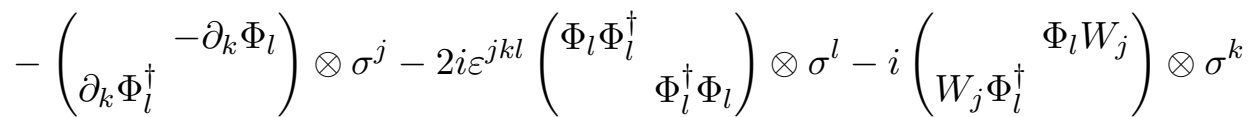

$$
\begin{aligned}
& +i\left(W_{k} \Phi_{l}^{\dagger} \Phi_{l} W_{k}\right) \otimes \sigma^{j}+i\left(\Phi_{l}^{\dagger} \sigma^{3} \sigma^{3} \Phi_{l}\right) \otimes\left(A_{j} \sigma^{k}-A_{k} \sigma^{j}\right) .
\end{aligned}
$$

so that

$$
\begin{aligned}
\kappa^{-1} \operatorname{Tr}\left(\Omega_{j k} \Omega_{j k}\right)= & -12 F_{j k} F_{j k}-2 \operatorname{Tr}\left(W_{j k} W_{j k}\right)-96 \operatorname{Tr}\left(\Phi_{l}^{\dagger} \Phi_{l}\right)^{2} \\
& -16 \operatorname{Tr}\left[\left(\partial_{j} \Phi_{l}^{\dagger}\right)\left(\partial_{j} \Phi_{l}\right)\right]-16 \operatorname{Tr}\left(W_{j}^{2} \Phi_{l}^{\dagger} \Phi_{l}\right)-16 A_{j}^{2} \operatorname{Tr}\left(\Phi_{l}^{\dagger} \Phi_{l}\right) \\
& +32 A_{j} \operatorname{Tr}\left(\Phi_{l} W_{j} \Phi_{l}^{\dagger} \sigma^{3}\right)-16 i \operatorname{Tr}\left[\left(\left(\partial_{j} \Phi_{l}^{\dagger}\right) \Phi_{l}-\Phi_{l}^{\dagger}\left(\partial_{j} \Phi_{l}\right)\right) W_{j}\right] \\
& +16 i A_{j} \operatorname{Tr}\left[\left(\Phi_{l}\left(\partial_{j} \Phi_{l}^{\dagger}\right)-\left(\partial_{j} \Phi_{l}\right) \Phi_{l}^{\dagger}\right) \sigma^{3}\right] .
\end{aligned}
$$

Therefore,

$$
\begin{aligned}
\kappa^{-1} \operatorname{Tr}\left(\Omega^{2}\right)= & -12 F^{2}-2 \operatorname{Tr}\left(W^{2}\right)-24 A_{\mu} A_{\mu} \operatorname{Tr}\left(\Phi_{l}^{\dagger} \Phi_{l}\right)-24 \operatorname{Tr}\left(W_{\mu} W_{\mu} \Phi_{l}^{\dagger} \Phi_{l}\right) \\
& -192 \operatorname{Tr}\left(\Phi_{l}^{\dagger} \Phi_{l}\right)^{2}+48 A_{\mu} \operatorname{Tr}\left(\Phi_{l}^{\dagger} \sigma^{3} \Phi_{l} W_{\mu}\right)-24 \operatorname{Tr}\left[\left(\partial_{\mu} \Phi_{l}\right)^{\dagger}\left(\partial_{\mu} \Phi_{l}\right)\right] \\
& -24 i A_{\mu} \operatorname{Tr}\left[\left(\left(\partial_{\mu} \Phi_{l}\right) \Phi_{l}^{\dagger}-\Phi_{l}\left(\partial_{\mu} \Phi_{l}^{\dagger}\right)\right) \sigma^{3}\right] \\
& +24 i \operatorname{Tr}\left[\left(\Phi_{l}^{\dagger}\left(\partial_{\mu} \Phi_{l}\right)-\left(\partial_{\mu} \Phi_{l}^{\dagger}\right) \Phi_{l}\right) W_{\mu}\right],
\end{aligned}
$$

where the summation is performed over Euclidean indices.

As a result, in the leptonic sector we have

$$
\begin{aligned}
a_{2}= & \frac{3 \kappa}{4 \pi^{2}} \int d^{4} x \operatorname{Tr}\left(\Phi_{l}^{\dagger} \Phi_{l}\right), \\
a_{4}= & \frac{\kappa}{48 \pi^{2}} \int d^{4} x\left[6 \left(\operatorname{Tr}\left(\Phi_{l}^{\dagger} \Phi_{l}\right)^{2}-\operatorname{Tr}\left[\left(\partial_{\mu} \Phi_{l}^{\dagger}\right)\left(\partial_{\mu} \Phi_{l}\right)\right]-A^{2} \operatorname{Tr}\left(\Phi_{l}^{\dagger} \Phi_{l}\right)\right.\right. \\
& \quad-\operatorname{Tr}\left(W_{\mu} W_{\mu} \Phi_{l}^{\dagger} \Phi_{l}\right)-i A_{\mu} \operatorname{Tr}\left[\left(\left(\partial_{\mu} \Phi_{l}\right) \Phi_{l}^{\dagger}-\Phi_{l}\left(\partial_{\mu} \Phi_{l}^{\dagger}\right)\right) \sigma^{3}\right] \\
& \left.+i \operatorname{Tr}\left[\left(\Phi_{l}^{\dagger}\left(\partial_{\mu} \Phi_{l}\right)-\left(\partial_{\mu} \Phi_{l}^{\dagger}\right) \Phi_{l}\right) W_{\mu}\right]+2 A_{\mu} \operatorname{Tr}\left(\Phi_{l}^{\dagger} \sigma^{3} \Phi_{l} W_{\mu}\right)\right) \\
& \left.+6 F^{2}+\operatorname{Tr}\left(W^{2}\right)+6 \varepsilon^{j k l} F_{j k} F_{0 l}-3 \varepsilon^{j k l} \operatorname{Tr}\left(W_{j k} W_{0 l}\right)\right] .
\end{aligned}
$$

Using the parametrization from section 3.1 we can further write

$$
a_{2}=\frac{3 \kappa}{4 \pi^{2}}\left(\left|\Upsilon_{e}\right|^{2}+\left|\Upsilon_{\nu}\right|^{2}\right) \int d^{4} x|H|^{2},
$$

and

$$
\begin{aligned}
a_{4}=\frac{\kappa}{8 \pi^{2}} \int d^{4} x & {\left[\left(\left|\Upsilon_{\nu}\right|^{4}+\left|\Upsilon_{e}\right|^{4}\right)|H|^{4}-\left(\left|\Upsilon_{\nu}\right|^{2}+\left|\Upsilon_{e}\right|^{2}\right) \operatorname{Tr}\left|D_{\mu} H\right|^{2}\right.} \\
+ & \left.F^{2}+\frac{1}{6} \operatorname{Tr}\left(W^{2}\right)+\varepsilon^{j k l} F_{j k} F_{0 l}-\frac{1}{2} \varepsilon^{j k l} \operatorname{Tr}\left(W_{j k} W_{0 l}\right)\right] .
\end{aligned}
$$




\section{B.2 Quark sector}

For the quark sector, starting from (3.7), we get

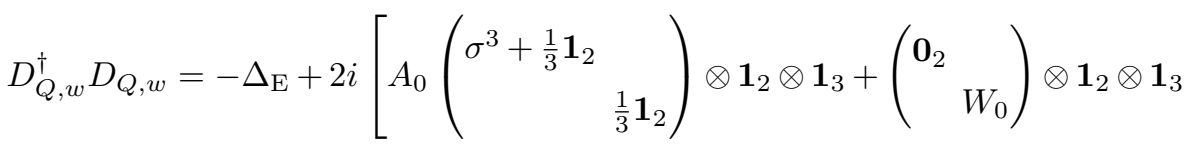

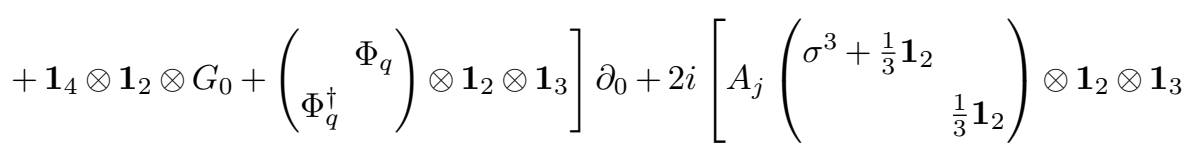

$$
\begin{aligned}
& \left.+\left(\begin{array}{cc}
\mathbf{0}_{2} & \\
& \\
& W_{j}
\end{array}\right) \otimes \mathbf{1}_{2} \otimes \mathbf{1}_{3}+\mathbf{1}_{4} \otimes \mathbf{1}_{2} \otimes G_{j}+\left(\begin{array}{cc} 
& -i \Phi_{q} \\
i \Phi_{q}^{\dagger} &
\end{array}\right) \otimes \sigma^{j} \otimes \mathbf{1}_{3}\right] \partial_{j} \\
& +i\left(\partial_{0} A_{0}\right)\left(\begin{array}{ccc}
\sigma^{3}+\frac{1}{3} \mathbf{1}_{2} & \\
& \frac{1}{3} \mathbf{1}_{2}
\end{array}\right) \otimes \mathbf{1}_{2} \otimes \mathbf{1}_{3}+i\left(\partial_{j} A_{k}\right)\left(\begin{array}{ccc}
\sigma^{3}+\frac{1}{3} \mathbf{1}_{2} & \\
& \frac{1}{3} \mathbf{1}_{2}
\end{array}\right) \otimes \sigma^{j} \sigma^{k} \otimes \mathbf{1}_{3} \\
& +A_{0}^{2}\left(\begin{array}{ll}
\frac{2}{3} \sigma^{3}+\frac{10}{9} \mathbf{1}_{2} & \\
& \frac{1}{9} \mathbf{1}_{2}
\end{array}\right) \otimes \mathbf{1}_{2} \otimes \mathbf{1}_{3}+A_{j} A_{k}\left(\begin{array}{ll}
\frac{2}{3} \sigma^{3}+\frac{10}{9} \mathbf{1}_{2} & \\
& \frac{1}{9} \mathbf{1}_{2}
\end{array}\right) \otimes \sigma^{j} \sigma^{k} \otimes \mathbf{1}_{3}
\end{aligned}
$$

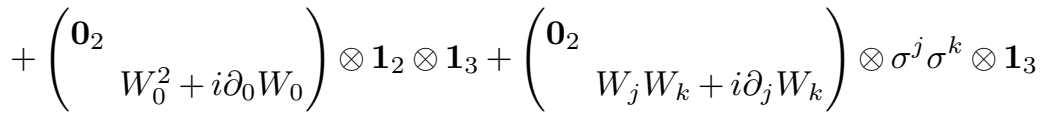

$$
\begin{aligned}
& +\mathbf{1}_{4} \otimes \mathbf{1}_{2} \otimes\left(G_{0}^{2}+i \partial_{0} G_{0}\right)+\mathbf{1}_{4} \otimes \sigma^{j} \sigma^{k} \otimes\left(G_{j} G_{k}+i \partial_{j} G_{k}\right)
\end{aligned}
$$

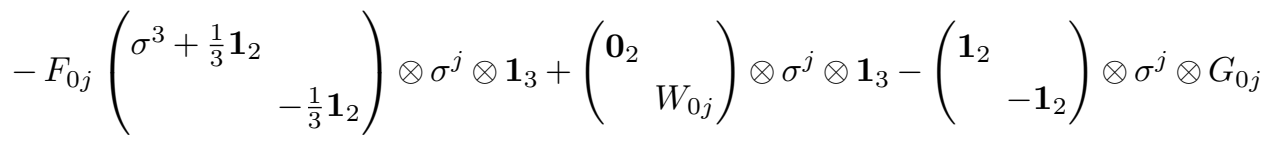

$$
\begin{aligned}
& +\frac{2}{3}\left(\begin{array}{ll}
\mathbf{0}_{2} & \\
& A_{0} W_{0}+A_{j} W_{j}
\end{array}\right) \otimes \mathbf{1}_{2} \otimes \mathbf{1}_{3}+2\left(\begin{array}{cc}
\sigma^{3}+\frac{1}{3} \mathbf{1}_{2} & \\
& \\
& \frac{1}{3} \mathbf{1}_{2}
\end{array}\right) \otimes \mathbf{1}_{2} \otimes\left(A_{0} G_{0}+A_{j} G_{j}\right)
\end{aligned}
$$

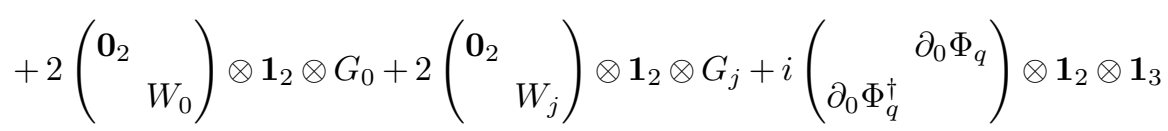

$$
\begin{aligned}
& +\left(\partial_{j} \Phi_{q}^{\dagger}{ }^{\partial_{j} \Phi_{q}}\right) \otimes \sigma^{j} \otimes \mathbf{1}_{3}+A_{0}\left(\begin{array}{c}
\sigma^{3} \Phi_{q}+\frac{2}{3} \Phi_{q} \\
\Phi_{q}^{\dagger} \sigma^{3}+\frac{2}{3} \Phi_{q}^{\dagger}
\end{array}\right) \otimes \mathbf{1}_{2} \otimes \mathbf{1}_{3} \\
& -i A_{j}\left(\begin{array}{c}
\sigma^{3} \Phi_{q}+\frac{2}{3} \Phi_{q} \\
-\Phi_{q}^{\dagger} \sigma^{3}-\frac{2}{3} \Phi_{q}^{\dagger}
\end{array}\right) \otimes \sigma^{j} \otimes \mathbf{1}_{3}+\left(\begin{array}{l}
W_{0} \Phi_{q}^{\dagger} \\
\Phi_{q} W_{0}
\end{array}\right) \otimes \mathbf{1}_{2} \otimes \mathbf{1}_{3} \\
& +i\left(W_{j} \Phi_{q}^{\dagger}-\Phi_{q} W_{j}\right) \otimes \sigma^{j} \otimes \mathbf{1}_{3}+2\left(\begin{array}{l}
\Phi_{q} \\
\Phi_{q}^{\dagger}
\end{array}\right) \otimes \mathbf{1}_{2} \otimes G_{0}
\end{aligned}
$$

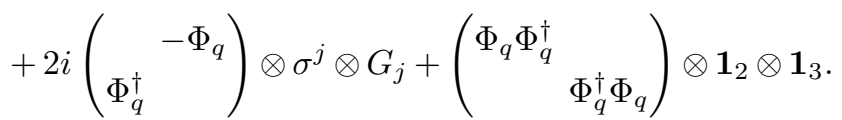


In this case we therefore have

$$
\begin{aligned}
& E=\frac{1}{2} F_{j k} \varepsilon^{j k l}\left(\begin{array}{ccc}
\sigma^{3}+\frac{1}{3} \mathbf{1}_{2} & \\
& \frac{1}{3} \mathbf{1}_{2}
\end{array}\right) \otimes \sigma^{l} \otimes \mathbf{1}_{3}+F_{0 j}\left(\begin{array}{lll}
\sigma^{3}+\frac{1}{3} \mathbf{1}_{2} & \\
& & \\
& & -\frac{1}{3} \mathbf{1}_{2}
\end{array}\right) \otimes \sigma^{j} \otimes \mathbf{1}_{3} \\
& +\frac{1}{2} \varepsilon^{j k l}\left(\begin{array}{cc}
\mathbf{0}_{2} & \\
& \\
& W_{j k}
\end{array}\right) \otimes \sigma^{l} \otimes \mathbf{1}_{3}-\left(\begin{array}{cc}
\mathbf{0}_{2} & \\
& W_{0 j}
\end{array}\right) \otimes \sigma^{j} \otimes \mathbf{1}_{3} \\
& +\frac{1}{2} \varepsilon^{j k l} \mathbf{1}_{4} \otimes \sigma^{l} \otimes G_{j k}+\left(\begin{array}{cc}
\mathbf{1}_{2} & \\
& -\mathbf{1}_{2}
\end{array}\right) \otimes \sigma^{j} \otimes G_{0 j} \\
& +3\left(\begin{array}{cc}
\Phi_{q} \Phi_{q}^{\dagger} & \\
& \Phi_{q}^{\dagger} \Phi_{q}
\end{array}\right) \otimes \mathbf{1}_{2} \otimes \mathbf{1}_{3} .
\end{aligned}
$$

Hence

$$
\operatorname{Tr}(E)=36 \kappa \operatorname{Tr}\left(\Phi_{q}^{\dagger} \Phi_{q}\right)
$$

Furthermore, we have

$\operatorname{Tr}\left(E^{2}\right)=\frac{22}{3} F^{2}+3 \operatorname{Tr}\left(W^{2}\right)+4 \operatorname{Tr}\left(G^{2}\right)+12 \varepsilon^{j k l} F_{j k} F_{0 l}-6 \varepsilon^{j k l} \operatorname{Tr}\left(W_{j k} W_{0 l}\right)+108 \operatorname{Tr}\left(\Phi_{q}^{\dagger} \Phi_{q}\right)^{2}$.

Moreover,

$$
\begin{aligned}
\Omega_{0 j}= & -F_{0 j}\left(\begin{array}{cc}
\sigma^{3}+\frac{1}{3} \mathbf{1}_{2} & \\
& \frac{1}{3} \mathbf{1}_{2}
\end{array}\right) \otimes \mathbf{1}_{2} \otimes \mathbf{1}_{3}-i\left(\begin{array}{cc}
\mathbf{0}_{2} & \\
& W_{0 j}
\end{array}\right) \otimes \mathbf{1}_{2} \otimes \mathbf{1}_{3}-i \mathbf{1}_{4} \otimes \mathbf{1}_{2} \otimes G_{0 j} \\
& +i A_{0}\left(\begin{array}{cc}
\sigma^{3} \Phi_{q} \\
\Phi_{q}^{\dagger} \sigma^{3}
\end{array}\right) \otimes \sigma^{j} \otimes \mathbf{1}_{3}-i\left(\begin{array}{c}
\Phi_{q} W_{0} \\
W_{0} \Phi_{q}^{\dagger}
\end{array}\right) \otimes \sigma^{j} \otimes \mathbf{1}_{3} \\
& -A_{j}\left(\begin{array}{cc}
\Phi_{q}^{\dagger} \sigma^{3} & -\sigma^{3} \Phi_{q} \\
W_{j} \Phi_{q}^{\dagger} & -\Phi_{q} W_{j}
\end{array}\right) \otimes \mathbf{1}_{2} \otimes \mathbf{1}_{2} \otimes \mathbf{1}_{3} \\
& -2 i\left(\begin{array}{cc}
\Phi_{q} \Phi_{q}^{\dagger} & \\
& -\Phi_{q}^{\dagger} \Phi_{q}
\end{array}\right) \otimes \sigma^{j} \otimes \mathbf{1}_{3}+i\left(\begin{array}{c}
\partial_{j} \Phi_{q} \\
\partial_{j} \Phi_{q}^{\dagger}
\end{array}\right) \otimes \mathbf{1}_{2} \otimes \mathbf{1}_{3} \\
& +\left(\begin{array}{rl}
\partial_{0} \Phi_{q}^{\dagger} & -\partial_{0} \Phi_{q}
\end{array}\right) \otimes \sigma^{j} \otimes \mathbf{1}_{3},
\end{aligned}
$$

hence

$$
\begin{aligned}
\kappa^{-1} \operatorname{Tr}\left(\Omega_{0 j} \Omega_{0 j}\right)= & -\frac{44}{3} F_{0 j} F_{0 j}-6 \operatorname{Tr}\left(W_{0 j} W_{0 j}\right)-8 \operatorname{Tr}\left(G_{0 j} G_{0 j}\right)-36 A_{0}^{2} \operatorname{Tr}\left(\Phi_{q}^{\dagger} \Phi_{q}\right) \\
& -36 \operatorname{Tr}\left(W_{0}^{2} \Phi_{q}^{\dagger} \Phi_{q}\right)-12 A_{j}^{2} \operatorname{Tr}\left(\Phi_{q}^{\dagger} \Phi_{q}\right)-12 \operatorname{Tr}\left(W_{j}^{2} \Phi_{q}^{\dagger} \Phi_{q}\right)-144 \operatorname{Tr}\left(\Phi_{q}^{\dagger} \Phi_{q}\right)^{2} \\
& +72 A_{0} \operatorname{Tr}\left(\Phi_{q}^{\dagger} \sigma^{3} \Phi_{q} W_{0}\right)+24 A_{j} \operatorname{Tr}\left(\Phi_{q}^{\dagger} \sigma^{3} \Phi_{q} W_{j}\right)-12 \operatorname{Tr}\left[\left(\partial_{j} \Phi_{q}\right)^{\dagger}\left(\partial_{j} \Phi_{q}\right)\right] \\
& -36 \operatorname{Tr}\left[\left(\partial_{0} \Phi_{q}\right)^{\dagger}\left(\partial_{0} \Phi_{q}\right)\right]-36 i A_{0} \operatorname{Tr}\left[\left(\left(\partial_{0} \Phi_{q}\right) \Phi_{q}^{\dagger}-\Phi_{q}\left(\partial_{0} \Phi_{q}\right)^{\dagger}\right) \sigma^{3}\right] \\
& -12 i A_{j} \operatorname{Tr}\left[\left(\left(\partial_{j} \Phi_{q}\right) \Phi_{q}^{\dagger}-\Phi_{q}\left(\partial_{j} \Phi_{q}\right)^{\dagger}\right) \sigma^{3}\right]+36 i \operatorname{Tr}\left[\left(\Phi_{q}^{\dagger}\left(\partial_{0} \Phi_{q}\right)-\left(\partial_{0} \Phi_{q}^{\dagger}\right) \Phi_{q}\right) W_{0}\right] \\
& +12 i \operatorname{Tr}\left[\left(\Phi_{q}^{\dagger}\left(\partial_{j} \Phi_{q}\right)-\left(\partial_{j} \Phi_{q}\right)^{\dagger} \Phi_{q}\right) W_{j}\right] .
\end{aligned}
$$


Moreover,

$$
\begin{aligned}
\Omega_{j k}= & -i F_{j k}\left(\begin{array}{cc}
\sigma^{3}+\frac{1}{3} \mathbf{1}_{2} & \\
& \frac{1}{3} \mathbf{1}_{2}
\end{array}\right) \otimes \mathbf{1}_{2} \otimes \mathbf{1}_{3}-i\left(\begin{array}{cc}
\mathbf{0}_{2} & \\
& W_{j k}
\end{array}\right) \otimes \mathbf{1}_{2} \otimes \mathbf{1}_{3}-i \mathbf{1}_{4} \otimes \mathbf{1}_{2} \otimes G_{j k}+ \\
& +\left(\begin{array}{c}
-\partial_{j} \Phi_{q} \\
\partial_{j} \Phi_{q}^{\dagger}
\end{array}\right) \otimes \sigma^{k} \otimes \mathbf{1}_{3}-\left(\begin{array}{c}
-\partial_{k} \Phi_{q} \\
\partial_{k} \Phi_{q}^{\dagger}
\end{array}\right) \otimes \sigma^{j} \otimes \mathbf{1}_{3} \\
& -2 i \varepsilon^{j k l}\left(\begin{array}{c}
\Phi_{q} \Phi_{q}^{\dagger} \\
\\
\end{array} \quad \Phi_{q}^{\dagger} \Phi_{q}\right) \otimes \sigma^{l} \otimes \mathbf{1}_{3}-i\left(\begin{array}{c}
W_{j} \Phi_{q}^{\dagger} \\
\Phi_{q} W_{j}
\end{array}\right) \otimes \sigma^{k} \otimes \mathbf{1}_{3} \\
& +i\left(\begin{array}{c}
\Phi_{q} W_{k} \\
W_{k} \Phi_{q}^{\dagger}
\end{array}\right) \otimes \sigma^{j} \otimes \mathbf{1}_{3}+i\left(\begin{array}{c}
\sigma^{3} \Phi_{q} \\
\Phi_{q}^{\dagger} \sigma^{3}
\end{array}\right) \otimes\left(A_{j} \sigma^{k}-A_{k} \sigma^{j}\right) \otimes \mathbf{1}_{3},
\end{aligned}
$$

and

$$
\begin{aligned}
\kappa^{-1} \operatorname{Tr}\left(\Omega_{j k} \Omega_{j k}\right)= & -\frac{44}{3} F_{j k} F_{j k}-6 \operatorname{Tr}\left(W_{j k} W_{j k}\right)-8 \operatorname{Tr}\left(G_{j k} G_{j k}\right)-288 \operatorname{Tr}\left(\Phi_{q}^{\dagger} \Phi_{q}\right)^{2} \\
& -48 \operatorname{Tr}\left[\left(\partial_{j} \Phi_{q}\right)^{\dagger}\left(\partial_{j} \Phi_{q}\right)\right]-48 \operatorname{Tr}\left(W_{j}^{2} \Phi_{q}^{\dagger} \Phi_{q}\right)-48 A_{j}^{2} \operatorname{Tr}\left(\Phi_{q}^{\dagger} \Phi_{q}\right) \\
& +96 A_{j} \operatorname{Tr}\left(\Phi_{q} W_{j} \Phi_{q}^{\dagger} \sigma^{3}\right)-48 i \operatorname{Tr}\left[\left(\left(\partial_{j} \Phi_{q}\right)^{\dagger} \Phi_{q}-\Phi_{q}^{\dagger}\left(\partial_{j} \Phi_{q}\right)\right) W_{j}\right] \\
& +48 i A_{j} \operatorname{Tr}\left[\left(\Phi_{q}\left(\partial_{j} \Phi_{q}\right)^{\dagger}-\left(\partial_{j} \Phi_{q}\right) \Phi_{q}^{\dagger}\right) \sigma^{3}\right] .
\end{aligned}
$$

Therefore

$$
\begin{aligned}
\kappa^{-1} \operatorname{Tr}\left(\Omega^{2}\right)= & -2\left(\frac{22}{3} F^{2}+3 \operatorname{Tr}\left(W^{2}\right)+4 \operatorname{Tr}\left(G^{2}\right)\right)-576 \operatorname{Tr}\left(\Phi_{q}^{\dagger} \Phi_{q}\right)^{2} \\
& -72 \operatorname{Tr}\left[\left(\partial_{\mu} \Phi_{q}\right)^{\dagger}\left(\partial_{\mu} \Phi_{q}\right)\right]-72 \operatorname{Tr}\left(W_{\mu} W_{\mu} \Phi_{q}^{\dagger} \Phi_{q}\right)-72 A_{\mu} A_{\mu} \operatorname{Tr}\left(\Phi_{q}^{\dagger} \Phi_{q}\right) \\
& +144 A_{\mu} \operatorname{Tr}\left(\Phi_{q}^{\dagger} \sigma^{3} \Phi_{q} W_{\mu}\right)-72 i A_{\mu} \operatorname{Tr}\left[\left(\left(\partial_{\mu} \Phi_{q}\right) \Phi_{q}^{\dagger}-\Phi_{q}\left(\partial_{\mu} \Phi_{q}\right)^{\dagger}\right) \sigma^{3}\right] \\
& +72 i \operatorname{Tr}\left[\left(\Phi_{q}^{\dagger}\left(\partial_{\mu} \Phi_{q}\right)-\left(\partial_{\mu} \Phi_{q}\right)^{\dagger} \Phi_{q}\right) W_{\mu}\right] .
\end{aligned}
$$

Expressing the coefficients $a_{2}$ and $a_{4}$ as in the section 3.1 we can further write

$$
a_{2}=\frac{3 \kappa}{4 \pi^{2}}\left(\left|\Upsilon_{e}\right|^{2}+\left|\Upsilon_{\nu}\right|^{2}\right) \int d^{4} x 3|H|^{2}
$$

and

$$
\begin{aligned}
a_{4}= & \frac{\kappa}{8 \pi^{2}} \int d^{4} x\left[\left(3\left|\Upsilon_{\nu}\right|^{4}+3\left|\Upsilon_{e}\right|^{4}\right)|H|^{4}-\left(3\left|\Upsilon_{\nu}\right|^{2}+3\left|\Upsilon_{e}\right|^{2}\right) \operatorname{Tr}\left|D_{\mu} H\right|^{2}\right. \\
& \left.+\frac{11}{9} F^{2}+\frac{1}{2} \operatorname{Tr}\left(W^{2}\right)+\frac{2}{3} \operatorname{Tr}\left(G^{2}\right)+3 \varepsilon^{j k l} F_{j k} F_{0 l}-\frac{3}{2} \varepsilon^{j k l} \operatorname{Tr}\left(W_{j k} W_{0 l}\right)\right] .
\end{aligned}
$$

\section{Acknowledgments}

AB acknowledges the hospitality of the Department of Mathematics of the Indiana University Bloomington during the Fulbright Junior Research Award scholarship funded by the Polish-U.S. Fulbright Commission. 
AB was partially supported by Faculty of Physics, Astronomy and Applied Computer Science of the Jagiellonian University under the MNS scheme: N17/MNS/000010.

AS and PZ acknowledge the support by NCN Grant 2020/37/B/ST1/01540.

The authors thank the referee for careful reading of the manuscript.

Open Access. This article is distributed under the terms of the Creative Commons Attribution License (CC-BY 4.0), which permits any use, distribution and reproduction in any medium, provided the original author(s) and source are credited.

\section{References}

[1] A. Gorokhovsky and E.V. Erp, Index theory and noncommutative geometry: a survey, in Advances in Noncommutative Geometry, A.H. Chamseddine, C. Consani, N. Higson, M. Khalkhali, H. Moscovici and G. Yu eds., Springer (2019).

[2] A. Connes, Noncommutative geometry and reality, J. Math. Phys. 36 (1995) 6194 [INSPIRE].

[3] A. Connes and J. Lott, Particle models and noncommutative geometry, Nucl. Phys. B Proc. Suppl. 18 (1991) 29 [INSPIRE].

[4] A. Connes, Gravity coupled with matter and the foundation of noncommutative geometry, Commun. Math. Phys. 182 (1996) 155 [hep-th/9603053] [INSPIRE].

[5] A. Connes, Noncommutative geometry, Academic Press, San Diego CA U.S.A. (1994).

[6] W.D. van Suijlekom, Noncommutative Geometry and Particle Physics, Springer (2015).

[7] C.A. Stephan, Almost-commutative geometries beyond the standard model, J. Phys. A 39 (2006) 9657 [hep-th/0509213] [INSPIRE].

[8] C.A. Stephan, Almost-commutative geometries beyond the standard model II: new colours, J. Phys. A 40 (2007) 9941 [arXiv:0706.0595] [InSPIRE].

[9] C.A. Stephan, New scalar fields in noncommutative geometry, Phys. Rev. D 79 (2009) 065013 [arXiv: 0901.4676] [INSPIRE].

[10] A. Devastato, F. Lizzi and P. Martinetti, Grand symmetry, spectral action and the Higgs mass, JHEP 01 (2014) 042 [arXiv: 1304.0415] [INSPIRE].

[11] A. Devastato, F. Lizzi and P. Martinetti, Higgs mass in noncommutative geometry, Fortsch. Phys. 62 (2014) 863 [arXiv: 1403.7567] [INSPIRE].

[12] A.H. Chamseddine, A. Connes and M. Marcolli, Gravity and the standard model with neutrino mixing, Adv. Theor. Math. Phys. 11 (2007) 991 [hep-th/0610241] [INSPIRE].

[13] M. Paschke, F. Scheck and A. Sitarz, Can (noncommutative) geometry accommodate leptoquarks?, Phys. Rev. D 59 (1999) 035003 [hep-th/9709009] [INSPIRE].

[14] L. Dąbrowski, F. D'Andrea and A. Sitarz, The Standard Model in noncommutative geometry: fundamental fermions as internal forms, Lett. Math. Phys. 108 (2018) 1323 [Erratum ibid. 109 (2019) 2585] [arXiv: 1703.05279] [INSPIRE].

[15] J.M. Gracia-Bondia, B. Iochum and T. Schucker, The standard model in noncommutative geometry and fermion doubling, Phys. Lett. B 416 (1998) 123 [hep-th/9709145] [INSPIRE].

[16] F. D'Andrea, M.A. Kurkov and F. Lizzi, Wick rotation and fermion doubling in noncommutative geometry, Phys. Rev. D 94 (2016) 025030 [arXiv:1605.03231] [InSPIRE]. 
[17] F. Lizzi, G. Mangano, G. Miele and G. Sparano, Fermion Hilbert space and fermion doubling in the noncommutative geometry approach to gauge theories, Phys. Rev. D 55 (1997) 6357 [hep-th/9610035] [INSPIRE].

[18] A. Bochniak and A. Sitarz, Spectral geometry for the standard model without fermion doubling, Phys. Rev. D 101 (2020) 075038 [arXiv:2001.02902] [INSPIRE].

[19] A.H. Chamseddine and A. Connes, Resilience of the spectral standard model, JHEP 09 (2012) 104 [arXiv:1208.1030] [INSPIRE].

[20] D.V. Vassilevich, Heat kernel expansion: user's manual, Phys. Rept. 388 (2003) 279 [hep-th/0306138] [INSPIRE].

[21] A.H. Chamseddine and A. Connes, The Uncanny Precision of the Spectral Action, Commun. Math. Phys. 293 (2010) 867 [arXiv:0812.0165] [INSPIRE].

[22] A. Bochniak and A. Sitarz, Finite pseudo-Riemannian spectral triples and the standard model, Phys. Rev. D 97 (2018) 115029 [arXiv: 1804.09482] [INSPIRE].

[23] S. Lord, A. Rennie and J.C. Várilly, Riemannian manifolds in noncommutative geometry, J. Geom. Phys. 62 (2012) 1611 [arXiv:1109.2196] [INSPIRE].

[24] A.H. Chamseddine and A. Connes, The Spectral Action Principle, Commun. Math. Phys. 186 (1997) 731 [hep-th/9606001] [INSPIRE].

[25] N.V. Dang and M. Wrochna, Complex powers of the wave operator and the spectral action on Lorentzian scattering spaces, arXiv:2012.00712 [INSPIRE].

[26] M. Eckstein and B. Iochum, Spectral Action in Noncommutative Geometry, in Springer Briefs in Mathematical Physics 27, Springer International Publishing (2018).

[27] P.O. Mazur and A. Staruszkiewicz, On the $\Theta$-term in electrodynamics, hep-th/9809205v1 [INSPIRE].

[28] P.F. Pérez and H.H. Patel, The electroweak vacuum angle, Phys. Lett. B 732 (2014) 241 [arXiv: 1402.6340] [INSPIRE].

[29] V.V. Khoze and D.L. Milne, Suppression of electroweak instanton processes in high-energy collisions, Int. J. Mod. Phys. A 36 (2021) 2150032 [arXiv:2011.07167] [inSPIRE].

[30] J. Boeijink and K. van den Dungen, On globally non-trivial almost-commutative manifolds, J. Math. Phys. 55 (2014) 103508 [arXiv:1405.5368] [InSPIRE].

[31] A. Bochniak and A. Sitarz, Stability of Friedmann-Lemaître-Robertson-Walker solutions in doubled geometries, Phys. Rev. D 103 (2021) 044041 [arXiv:2012.06401] [InSPIRE].

[32] A.H. Chamseddine, A. Connes and W.D. van Suijlekom, Beyond the spectral standard model: emergence of Pati-Salam unification, JHEP 11 (2013) 132 [arXiv:1304.8050] [INSPIRE].

[33] A. Bochniak, T.E. Williams and P. Zalecki, Pseudo-Riemannian structures in Pati-Salam models, JHEP 07 (2020) 072 [arXiv: 2002.04506] [INSPIRE]. 